\title{
Positive Solutions and Mann Iterations of a Fourth Order Nonlinear Neutral Delay Differential Equation
}

\author{
Zeqing Liu, ${ }^{1}$ Jingjing Zhu, ${ }^{2}$ Jeong Sheok Ume, ${ }^{3}$ and Shin Min Kang ${ }^{4}$ \\ ${ }^{1}$ Department of Mathematics, Liaoning Normal University, Dalian, Liaoning 116029, China \\ ${ }^{2}$ Dalian No. 39 Middle School Dalian, Liaoning 116001, China \\ ${ }^{3}$ Department of Mathematics, Changwon National University, Changwon 641-773, Republic of Korea \\ ${ }^{4}$ Department of Mathematics and RINS, Gyeongsang National University, Jinju 660-701, Republic of Korea
}

Correspondence should be addressed to Jeong Sheok Ume; jsume@changwon.ac.kr

Received 16 December 2013; Accepted 20 February 2014; Published 29 May 2014

Academic Editor: Allan Peterson

Copyright (C) 2014 Zeqing Liu et al. This is an open access article distributed under the Creative Commons Attribution License, which permits unrestricted use, distribution, and reproduction in any medium, provided the original work is properly cited.

\begin{abstract}
This paper deals with a fourth order nonlinear neutral delay differential equation. By using the Banach fixed point theorem, we establish the existence of uncountably many bounded positive solutions for the equation, construct several Mann iterative sequences with mixed errors for approximating these positive solutions, and discuss some error estimates between the approximate solutions and these positive solutions. Seven nontrivial examples are given.
\end{abstract}

\section{Introduction and Preliminaries}

The oscillation, nonoscillation, and existence of solutions for various kinds of second order and third order neutral delay differential equations have been extensively studied over the last decades; for example, see [1-12]. Elbert [2] and Huang [3] established a few oscillation and nonoscillation criteria for the second order linear differential equation

$$
x^{\prime \prime}(t)+q(t) x(t)=0, \quad t \geq 0,
$$

where $q \in C\left([0,+\infty), \mathbb{R}^{+}\right)$. Tang and Liu [9] studied the existence of bounded oscillation for the second order linear delay differential equation of unstable type

$$
x^{\prime \prime}(t)=p(t) x(t-\tau), \quad t \geq t_{0},
$$

where $\tau>0, p \in C\left(\left[t_{0},+\infty\right), \mathbb{R}^{+}\right)$, and $p(t) \quad \not \quad 0$ on any interval of length $\tau$. Using the Banach fixed point theorem, Kulenović and Hadžiomerspahić [4] deduced the existence of a nonoscillatory solution for the second order linear neutral delay differential equation with positive and negative coefficients

$$
\begin{gathered}
(x(t)+c x(t-\tau))^{\prime \prime}+Q_{1}(t) x\left(t-\sigma_{1}\right) \\
-Q_{2}(t) x\left(t-\sigma_{2}\right)=0, \quad t \geq t_{0},
\end{gathered}
$$

where $c \in \mathbb{R} \backslash\{-1,1\}, \tau>0, \sigma_{1}, \sigma_{2} \in[0,+\infty), Q_{1}$, $Q_{2} \in C\left(\left[t_{0},+\infty\right), \mathbb{R}^{+}\right)$. Lin [5] suggested a few sufficient conditions for oscillation and nonoscillation of the second order nonlinear neutral differential equation

$$
(x(t)+p(t) x(t-\tau))^{\prime \prime}+q(t) f(x(t-\sigma))=0, \quad t \geq t_{0},
$$

where $\tau>0, \sigma \geq 0, p \in C\left(\left[t_{0},+\infty\right), \mathbb{R}^{+}\right), q \in C\left(\mathbb{R}, \mathbb{R}^{+}\right)$, $f \in C(\mathbb{R}, \mathbb{R}), f$ is nondecreasing, and $x f(x)>0, x \neq 0$. Qin et al. [8] and Yang et al. [11] developed several oscillation criteria for the second order differential equation

$$
\begin{aligned}
& \left(r(t)(x(t)+p(t) x(t-\tau))^{\prime}\right)^{\prime} \\
& \quad+q(t) f(x(t-\delta))=0, \quad t \geq t_{0},
\end{aligned}
$$


where $\tau$ and $\delta$ are nonnegative constants, $r, p, q \epsilon$ $C\left(\left[t_{0},+\infty\right), \mathbb{R}\right)$, and $f \in C(\mathbb{R}, \mathbb{R})$. By utilizing Krasnoselskii's fixed point theorem, Zhou [12] discussed the existence of nonoscillatory solutions of the second order nonlinear neutral differential equation

$$
\begin{aligned}
& \left(r(t)(x(t)+p(t) x(t-\tau))^{\prime}\right)^{\prime} \\
& \quad+\sum_{i=1}^{m} Q_{i}(t) f_{i}\left(x\left(t-\sigma_{i}\right)\right)=0, \quad t \geq t_{0},
\end{aligned}
$$

where $m \geq 1$ is an integer, $\tau>0, \sigma_{i} \geq 0, r, p, Q_{i} \in$ $C\left(\left[t_{0},+\infty\right), \mathbb{R}\right)$, and $f_{i} \in C(\mathbb{R}, \mathbb{R})$ for $i \in\{1,2, \ldots, m\}$. Yu and Wang [10] studied the existence of a nonoscillatory solution for the second order nonlinear neutral delay differential equations with positive and negative coefficients

$$
\begin{gathered}
\left(r(t)(x(t)+P(t) x(t-\tau))^{\prime}\right)^{\prime}+Q_{1}(t) f\left(x\left(t-\sigma_{1}\right)\right) \\
-Q_{2}(t) g\left(x\left(t-\sigma_{2}\right)\right)=0, \quad t \geq t_{0},
\end{gathered}
$$

where $\tau>0, \sigma_{1}, \sigma_{2} \in[0,+\infty), P, Q_{1}, Q_{2}, r \in C\left(\left[t_{0},+\infty\right), \mathbb{R}\right)$, $f, g \in C(\mathbb{R}, \mathbb{R})$. Liu and Kang [7] investigated the existence of nonoscillatory solutions of the second order nonlinear neutral delay differential equation

$$
\begin{aligned}
(a(t) & \left.(x(t)+b(t) x(t-\tau))^{\prime}\right)^{\prime} \\
& +\left(h\left(t, x\left(h_{1}(t)\right), x\left(h_{2}(t)\right), \ldots, x\left(h_{k}(t)\right)\right)\right)^{\prime} \\
+ & f\left(t, x\left(f_{1}(t)\right), x\left(f_{2}(t)\right), \ldots, x\left(f_{k}(t)\right)\right)=g(t), \\
& t \geq t_{0},
\end{aligned}
$$

where $\tau>0, a, b, g \in C\left(\left[t_{0},+\infty\right), \mathbb{R}\right)$ with $a(t)>0$ for $t \geq t_{0}$, $h \in C^{1}\left(\left[t_{0},+\infty\right) \times \mathbb{R}^{k}, \mathbb{R}\right), f \in C\left(\left[t_{0},+\infty\right) \times \mathbb{R}^{k}, \mathbb{R}\right), h_{l} \in$ $C^{1}\left(\left[t_{0},+\infty\right), \mathbb{R}\right)$, and $f_{l} \in C\left(\left[t_{0},+\infty\right), \mathbb{R}\right)$ with

$$
\lim _{t \rightarrow+\infty} h_{l}(t)=\lim _{t \rightarrow+\infty} f_{l}(t)=+\infty, \quad l=1, \ldots, k
$$

Kang et al. [13] discussed the existence of nonoscillatory solutions of the third order nonlinear neutral delay differential equation

$$
\begin{aligned}
\left(\alpha(t)\left(\beta(t)(x(t)+p(t) x(t-\tau))^{\prime}\right)^{\prime}\right)^{\prime} & \\
+f\left(t, x\left(\sigma_{1}(t)\right), x\left(\sigma_{2}(t)\right), \ldots, x\left(\sigma_{n}(t)\right)\right) & =0 \\
t & \geq t_{0}
\end{aligned}
$$

where $n \geq 1$ is an integer, $\tau>0, \alpha, \beta \in C\left(\left[t_{0},+\infty\right), \mathbb{R}^{+} \backslash\{0\}\right)$, $p \in C\left(\left[t_{0},+\infty\right), \mathbb{R}\right)$, and $f \in C\left(\left[t_{0},+\infty\right) \times \mathbb{R}^{n}, \mathbb{R}\right)$.
Motivated by the papers mentioned above, in this paper, we investigate the following fourth order nonlinear neutral delay differential equation:

$$
\begin{aligned}
(\alpha(t) & \left.\left(\beta(t)\left(\gamma(t)(x(t)+p(t) x(t-\tau))^{\prime}\right)^{\prime}\right)^{\prime}\right)^{\prime} \\
& \\
& +\left(h\left(t, x\left(h_{1}(t)\right), \ldots, x\left(h_{k}(t)\right)\right)\right)^{\prime} \\
& +f\left(t, x\left(f_{1}(t)\right), x\left(f_{2}(t)\right), \ldots, x\left(f_{k}(t)\right)\right)=g(t), \\
& t \geq t_{0},
\end{aligned}
$$

where $k \in \mathbb{N}, \tau>0, \alpha, \beta, \gamma \in C\left(\left[t_{0},+\infty\right), \mathbb{R} \backslash\{0\}\right), p, g \in$ $C\left(\left[t_{0},+\infty\right), \mathbb{R}\right), h \in C^{1}\left(\left[t_{0},+\infty\right) \times \mathbb{R}^{k}, \mathbb{R}\right), f \in C\left(\left[t_{0},+\infty\right) \times\right.$ $\left.\mathbb{R}^{k}, \mathbb{R}\right), h_{l} \in C^{1}\left(\left[t_{0},+\infty\right), \mathbb{R}\right)$, and $f_{l} \in C\left(\left[t_{0},+\infty\right), \mathbb{R}\right)$ with

$$
\lim _{t \rightarrow+\infty} h_{l}(t)=\lim _{t \rightarrow+\infty} f_{l}(t)=+\infty, \quad l=1,2, \ldots, k
$$

Utilizing the contraction mapping principle, we show the existence of uncountably many bounded positive solutions for (11), construct a few Mann type iterative schemes with mixed errors for these positive solutions, and discuss error estimates between the approximate solutions and the bounded positive solutions. Seven nontrivial examples are considered to illustrate our results.

Throughout this paper, we assume that $\mathbb{R}=(-\infty,+\infty)$, $\mathbb{N}$ denotes the set of positive integers, $\mathbb{N}_{0}=\{0\} \cup \mathbb{N}$, and

$$
a=\min \left\{t_{0}-\tau, \inf \left\{h_{l}(t), f_{l}(t): t \in\left[t_{0},+\infty\right), 1 \leq l \leq k\right\}\right\} .
$$

By a solution of (11), we mean a function $x \in$ $C([a,+\infty), \mathbb{R})$ for some $T \geq t_{0}+|a|+\tau$, such that $\alpha(t)\left(\beta(t)\left(\gamma(t)(x(t)+p(t) x(t-\tau))^{\prime}\right)^{\prime}\right)^{\prime}$ is continuously differentiable in $[T,+\infty)$ and (11) is satisfied for $t \geq T$.

Let $C B([a,+\infty), \mathbb{R})$ denote the Banach space of all continuous and bounded functions on $[a,+\infty)$ with the norm $\|x\|=\sup _{t \geq a}|x(t)|$ for each $x \in C B([a,+\infty), \mathbb{R})$ and

$$
\begin{array}{r}
A(N, M)=\{x \in C B([a,+\infty), \mathbb{R}): N \leq x(t) \leq M, t \geq a\} \\
\text { for } M>N>0 .
\end{array}
$$

It is easy to see that $A(N, M)$ is a bounded closed and convex subset of $C B([a,+\infty), \mathbb{R})$.

The following lemma plays an important role in this paper. 
Lemma 1 (see [6]). Let $\left\{\alpha_{n}\right\}_{n \in \mathbb{N}_{0}},\left\{\beta_{n}\right\}_{n \in \mathbb{N}_{0}},\left\{\gamma_{n}\right\}_{n \in \mathbb{N}_{0}}$, and $\left\{t_{n}\right\}_{n \in \mathbb{N}_{0}}$ be four nonnegative real sequences satisfying the inequality

$$
\alpha_{n+1} \leq\left(1-t_{n}\right) \alpha_{n}+t_{n} \beta_{n}+\gamma_{n}, \quad n \in \mathbb{N}_{0},
$$

where $\left\{t_{n}\right\}_{n \in \mathbb{N}_{0}} \subset[0,1], \sum_{n=0}^{\infty} t_{n}=+\infty, \lim _{n \rightarrow \infty} \beta_{n}=0$, and $\sum_{n=0}^{\infty} \gamma_{n}<+\infty$. Then $\lim _{n \rightarrow \infty} \alpha_{n}=0$.

\section{Uncountably Many Bounded Positive Solutions and Iterative Approximations}

Now we study the solvability of (11).

Theorem 2. Assume that there exist constants $M, N, c_{1}, c_{2}$, and $T_{0} \geq t_{0}$ and functions $Q_{1}, Q_{2}, R_{1}$, and $R_{2} \in$ $C\left(\left[t_{0},+\infty\right), R^{+}\right)$satisfying

$$
\begin{array}{r}
\left|f\left(t, u_{1}, u_{2}, \ldots, u_{k}\right)-f\left(t, \bar{u}_{1}, \bar{u}_{2}, \ldots, \bar{u}_{k}\right)\right| \\
\leq Q_{1}(t) \max \left\{\left|u_{l}-\bar{u}_{l}\right|: 1 \leq l \leq k\right\}, \\
\left|h\left(t, u_{1}, u_{2}, \ldots, u_{k}\right)-h\left(t, \bar{u}_{1}, \bar{u}_{2}, \ldots, \bar{u}_{k}\right)\right| \\
\leq Q_{2}(t) \max \left\{\left|u_{l}-\bar{u}_{l}\right|: 1 \leq l \leq k\right\}
\end{array}
$$$$
\text { for } t \in\left[t_{0},+\infty\right), \quad u_{l}, \bar{u}_{l} \in[N, M], \quad 1 \leq l \leq k \text {; }
$$$$
\left|f\left(t, u_{1}, u_{2}, \ldots, u_{k}\right)\right| \leq R_{1}(t),
$$$$
\left|h\left(t, u_{1}, u_{2}, \ldots, u_{k}\right)\right| \leq R_{2}(t),
$$$$
\text { for } t \in\left[t_{0},+\infty\right), u_{l} \in[N, M] \text {, }
$$$$
1 \leq l \leq k
$$

$$
\begin{aligned}
& \int_{t_{0}}^{+\infty} \int_{w}^{+\infty} \int_{v}^{+\infty} \frac{1}{|\alpha(u) \beta(v) \gamma(w)|} \\
& \quad \times \max \left\{Q_{2}(u), R_{2}(u)\right\} d u d v d w<+\infty, \\
& \int_{t_{0}}^{+\infty} \int_{w}^{+\infty} \int_{v}^{+\infty} \int_{u}^{+\infty} \frac{1}{|\alpha(u) \beta(v) \gamma(w)|} \\
& \quad \times \max \left\{Q_{1}(s), R_{1}(s),|g(s)|\right\} d s d u d v d w<+\infty ;
\end{aligned}
$$

$\min \left\{c_{1}, c_{2}\right\} \geq 0, \quad c_{1}+c_{2}<1, \quad 0<N<\left(1-c_{1}-c_{2}\right) M$;

$$
-c_{2} \leq p(t) \leq c_{1}, \quad t \geq T_{0} .
$$

Then,

(a) for any $L \in\left(N+c_{1} M,\left(1-c_{2}\right) M\right)$, there exist $\theta \in$ $(0,1)$ and $T>t_{0}+|a|+\tau+\left|T_{0}\right|$ such that for each $x_{0} \in A(N, M)$, the Mann iterative sequence $\left\{x_{m}\right\}_{m \in \mathbb{N}_{0}}$ with mixed errors generated by the scheme

$$
\begin{aligned}
& \left\{\begin{array}{c}
\left(1-\alpha_{m}-\beta_{m}\right) x_{m}(t)+\alpha_{m} \\
\times\left\{L-p(t) x_{m}(t-\tau)\right.
\end{array}\right. \\
& +\int_{t}^{+\infty} \int_{w}^{+\infty} \int_{v}^{+\infty} \frac{1}{\alpha(u) \beta(v) \gamma(w)} \\
& \times h\left(u, x_{m}\left(h_{1}(u)\right), \ldots, x_{m}\left(h_{k}(u)\right)\right) d u d v d w \\
& -\int_{t}^{+\infty} \int_{w}^{+\infty} \int_{v}^{+\infty} \int_{u}^{+\infty} \frac{1}{\alpha(u) \beta(v) \gamma(w)} \\
& \times\left[f\left(s, x_{m}\left(f_{1}(s)\right), \ldots, x_{m}\left(f_{k}(s)\right)\right)\right. \\
& -g(s)] d s d u d v d w\} \\
& x_{m+1}(t)=\left\{\begin{array}{l}
+\beta_{m} \gamma_{m}(t), \quad t \geq T, m \in \mathbb{N}_{0}, \\
\left(1-\alpha_{m}-\beta_{m}\right) x_{m}(T)+\alpha_{m}
\end{array}\right. \\
& \left(1-\alpha_{m}-\beta_{m}\right) x_{m}(T)+\alpha_{m} \\
& \times\left\{L-p(T) x_{m}(T-\tau)\right. \\
& +\int_{T}^{+\infty} \int_{w}^{+\infty} \int_{v}^{+\infty} \frac{1}{\alpha(u) \beta(v) \gamma(w)} \\
& \times h\left(u, x_{m}\left(h_{1}(u)\right), \ldots\right. \text {, } \\
& \left.x_{m}\left(h_{k}(u)\right)\right) d u d v d w \\
& -\int_{T}^{+\infty} \int_{w}^{+\infty} \int_{v}^{+\infty} \int_{u}^{+\infty} \frac{1}{\alpha(u) \beta(v) \gamma(w)} \\
& \times\left[f\left(s, x_{m}\left(f_{1}(s)\right), \ldots, x_{m}\left(f_{k}(s)\right)\right)\right. \\
& -g(s)] d s d u d v d w\} \\
& +\beta_{m} \gamma_{m}(T), \quad a \leq t<T, m \in \mathbb{N}_{0}
\end{aligned}
$$

converges to a bounded positive solution $x \in A(N, M)$ of (11) and has the following error estimate:

$$
\left\|x_{m+1}-x\right\| \leq\left(1-(1-\theta) \alpha_{m}\right)\left\|x_{m}-x\right\|+2 M \beta_{m}, \quad m \in \mathbb{N}_{0},
$$

where $\left\{\gamma_{m}\right\}_{m \in \mathbb{N}_{0}}$ is an arbitrary sequence in $A(N, M)$ and $\left\{\alpha_{m}\right\}_{m \in \mathbb{N}_{0}}$ and $\left\{\beta_{m}\right\}_{m \in \mathbb{N}_{0}}$ are any sequences in $[0,1]$ such that

$$
\sum_{m=0}^{\infty} \alpha_{m}=+\infty,
$$

$\sum_{m=0}^{\infty} \beta_{m}<+\infty$ or there exists a sequence

$$
\begin{gathered}
\left\{\xi_{m}\right\}_{m \in \mathbb{N}_{0}} \subseteq[0,+\infty) \text { satisfying } \\
\beta_{m}=\xi_{m} \alpha_{m}, m \in \mathbb{N}_{0}, \lim _{m \rightarrow \infty} \xi_{m}=0 ;
\end{gathered}
$$

(b) equation (11) possesses uncountably many bounded positive solutions in $A(N, M)$. 
Proof. Firstly, we show that (a) holds. Let $L \in\left(N+c_{1} M,(1-\right.$ $\left.\left.c_{2}\right) M\right)$. It follows from (18) and (19) that there exist $\theta \in(0,1)$ and $T>t_{0}+|a|+\tau+\left|T_{0}\right|$ satisfying

$$
\begin{aligned}
\theta= & c_{1}+c_{2}+\int_{T}^{+\infty} \int_{w}^{+\infty} \int_{v}^{+\infty} \frac{Q_{2}(u)}{|\alpha(u) \beta(v) \gamma(w)|} d u d v d w \\
& +\int_{T}^{+\infty} \int_{w}^{+\infty} \int_{v}^{+\infty} \int_{u}^{+\infty} \frac{Q_{1}(s)}{|\alpha(u) \beta(v) \gamma(w)|} d s d u d v d w \\
& \int_{T}^{+\infty} \int_{w}^{+\infty} \int_{v}^{+\infty} \frac{R_{2}(u)}{|\alpha(u) \beta(v) \gamma(w)|} d u d v d w \\
& +\int_{T}^{+\infty} \int_{w}^{+\infty} \int_{v}^{+\infty} \int_{u}^{+\infty} \frac{R_{1}(s)+|g(s)|}{|\alpha(u) \beta(v) \gamma(w)|} d s d u d v d w \\
< & \min \left\{\left(1-c_{2}\right) M-L, L-N-c_{1} M\right\} .
\end{aligned}
$$

Define a mapping $S_{L}: A(N, M) \rightarrow C B([a,+\infty), \mathbb{R})$ by

$$
S_{L} x(t)=\left\{\begin{aligned}
& L- p(t) x(t-\tau) \\
&+\int_{t}^{+\infty} \int_{w}^{+\infty} \int_{v}^{+\infty} \frac{1}{\alpha(u) \beta(v) \gamma(w)} \\
& \times h\left(u, x\left(h_{1}(u)\right), \ldots, x\left(h_{k}(u)\right)\right) d u d v d w \\
&-\int_{t}^{+\infty} \int_{w}^{+\infty} \int_{v}^{+\infty} \int_{u}^{+\infty} \frac{1}{\alpha(u) \beta(v) \gamma(w)} \\
& \times\left[f\left(s, x\left(f_{1}(s)\right), \ldots, x\left(f_{k}(s)\right)\right)\right. \\
&-g(s)] d s d u d v d w, \\
& t \geq T, x \in A(N, M), \\
& S_{L} x(T), a \leq t<T, x \in A(N, M) .
\end{aligned}\right.
$$

Obviously, $S_{L} x$ is continuous for each $x \in A(N, M)$. Combining (16), (17), (19), (20), and (25)-(27), we derive that for $x, y \in A(N, M)$ and $t \geq T$

$$
\begin{aligned}
& \left|S_{L} x(t)-S_{L} y(t)\right| \\
& \leq|p(t)||x(t-\tau)-y(t-\tau)| \\
& \quad+\int_{t}^{+\infty} \int_{w}^{+\infty} \int_{v}^{+\infty} \frac{1}{|\alpha(u) \beta(v) \gamma(w)|} \\
& \quad \times \mid h\left(u, x\left(h_{1}(u)\right), \ldots, x\left(h_{k}(u)\right)\right) \\
& \quad-h\left(u, y\left(h_{1}(u)\right), \ldots, y\left(h_{k}(u)\right)\right) \mid d u d v d w \\
& +\int_{t}^{+\infty} \int_{w}^{+\infty} \int_{v}^{+\infty} \int_{u}^{+\infty} \frac{1 \alpha(u) \beta(v) \gamma(w) \mid}{\mid f\left(s, x\left(f_{1}(s)\right), \ldots, x\left(f_{k}(s)\right)\right)} \\
& \quad \times\left|f\left(s, y\left(f_{1}(s)\right), \ldots, y\left(f_{k}(s)\right)\right)\right| d s d u d v d w
\end{aligned}
$$

$$
\begin{aligned}
\leq & \left(c_{1}+c_{2}\right)\|x-y\|+\|x-y\| \\
& \times \int_{t}^{+\infty} \int_{w}^{+\infty} \int_{v}^{+\infty} \frac{Q_{2}(u)}{|\alpha(u) \beta(v) \gamma(w)|} d u d v d w \\
& +\|x-y\| \\
& \times \int_{t}^{+\infty} \int_{w}^{+\infty} \int_{v}^{+\infty} \int_{u}^{+\infty} \frac{Q_{1}(s)}{|\alpha(u) \beta(v) \gamma(w)|} d s d u d v d w \\
\leq & {\left[c_{1}+c_{2}+\int_{T}^{+\infty} \int_{w}^{+\infty} \int_{v}^{+\infty} \frac{Q_{2}(u)}{|\alpha(u) \beta(v) \gamma(w)|} d u d v d w\right.} \\
& \left.+\int_{T}^{+\infty} \int_{w}^{+\infty} \int_{v}^{+\infty} \int_{u}^{+\infty} \frac{Q_{1}(s)}{|\alpha(u) \beta(v) \gamma(w)|} d s d u d v d w\right] \\
& \times\|x-y\| \\
= & \theta\|x-y\|,
\end{aligned}
$$

$S_{L} x(t)$

$$
\begin{aligned}
& =L-p(t) x(t-\tau) \\
& +\int_{t}^{+\infty} \int_{w}^{+\infty} \int_{v}^{+\infty} \frac{1}{\alpha(u) \beta(v) \gamma(w)} \\
& \times h\left(u, x\left(h_{1}(u)\right), \ldots, x\left(h_{k}(u)\right)\right) d u d v d w \\
& -\int_{t}^{+\infty} \int_{w}^{+\infty} \int_{v}^{+\infty} \int_{u}^{+\infty} \frac{1}{\alpha(u) \beta(v) \gamma(w)} \\
& \times\left[f\left(s, x\left(f_{1}(s)\right), \ldots, x\left(f_{k}(s)\right)\right)-g(s)\right] d s d u d v d w \\
& \leq L+c_{2} M \\
& +\int_{t}^{+\infty} \int_{w}^{+\infty} \int_{v}^{+\infty} \frac{1}{|\alpha(u) \beta(v) \gamma(w)|} \\
& \times\left|h\left(u, x\left(h_{1}(u)\right), \ldots, x\left(h_{k}(u)\right)\right)\right| d u d v d w \\
& +\int_{t}^{+\infty} \int_{w}^{+\infty} \int_{v}^{+\infty} \int_{u}^{+\infty} \frac{1}{|\alpha(u) \beta(v) \gamma(w)|} \\
& \times\left[\left|f\left(s, x\left(f_{1}(s)\right), \ldots, x\left(f_{k}(s)\right)\right)\right|+|g(s)|\right] d s d u d v d w \\
& \leq L+c_{2} M \\
& +\int_{T}^{+\infty} \int_{w}^{+\infty} \int_{v}^{+\infty} \frac{R_{2}(u)}{|\alpha(u) \beta(v) \gamma(w)|} d u d v d w \\
& +\int_{T}^{+\infty} \int_{w}^{+\infty} \int_{v}^{+\infty} \int_{u}^{+\infty} \frac{R_{1}(s)+|g(s)|}{|\alpha(u) \beta(v) \gamma(w)|} d s d u d v d w \\
& <L+c_{2} M+\min \left\{\left(1-c_{2}\right) M-L, L-N-c_{1} M\right\} \\
& \leq M \text {, } \\
& S_{L} x(t) \\
& \geq L-c_{1} M
\end{aligned}
$$




$$
\begin{aligned}
& -\int_{t}^{+\infty} \int_{w}^{+\infty} \int_{v}^{+\infty} \frac{1}{|\alpha(u) \beta(v) \gamma(w)|} \\
& \times\left|h\left(u, x\left(h_{1}(u)\right), \ldots, x\left(h_{k}(u)\right)\right)\right| d u d v d w \\
& -\int_{t}^{+\infty} \int_{w}^{+\infty} \int_{v}^{+\infty} \int_{u}^{+\infty} \frac{1}{|\alpha(u) \beta(v) \gamma(w)|} \\
& \times\left|\left[f\left(s, x\left(f_{1}(s)\right), \ldots, x\left(f_{k}(s)\right)\right)-g(s)\right]\right| d s d u d v d w \\
\geq & L-c_{1} M \\
& -\int_{T}^{+\infty} \int_{w}^{+\infty} \int_{v}^{+\infty} \frac{R_{2}(u)}{|\alpha(u) \beta(v) \gamma(w)|} d u d v d w \\
& -\int_{T}^{+\infty} \int_{w}^{+\infty} \int_{v}^{+\infty} \int_{u}^{+\infty} \frac{R_{1}(s)+|g(s)|}{|\alpha(u) \beta(v) \gamma(w)|} d s d u d v d w \\
> & L-c_{1} M-\min \left\{\left(1-c_{2}\right) M-L, L-N-c_{1} M\right\} \\
\geq & N,
\end{aligned}
$$

which mean that

$$
\begin{gathered}
\left\|S_{L} x-S_{L} y\right\| \leq \theta\|x-y\|, \quad x, y \in A(N, M), \\
S_{L}(A(N, M)) \subseteq A(N, M) .
\end{gathered}
$$

That is, $S_{L}$ is a contraction mapping in $A(N, M)$ and has a unique fixed point $x \in A(N, M)$, which is a bounded positive solution of (11). By virtue of (21), (27), and (29), we get that for any $m \in \mathbb{N}_{0}$ and $t \geq T$

$$
\begin{aligned}
& \mid x_{m+1}(t)-x(t) \mid \\
&=\mid\left(1-\alpha_{m}-\beta_{m}\right) x_{m}(t)+\alpha_{m} \\
& \times\left\{L-p(t) x_{m}(t-\tau)\right. \\
&+\int_{t}^{+\infty} \int_{w}^{+\infty} \int_{v}^{+\infty} \frac{1}{\alpha(u) \beta(v) \gamma(w)} \\
& \times h\left(u, x_{m}\left(h_{1}(u)\right), \ldots, x_{m}\left(h_{k}(u)\right)\right) d u d v d w \\
& \quad-\int_{t}^{+\infty} \int_{w}^{+\infty} \int_{v}^{+\infty} \int_{u}^{+\infty} \frac{1}{\alpha(u) \beta(v) \gamma(w)} \\
& \times\left[f\left(s, x_{m}\left(f_{1}(s)\right), \ldots, x_{m}\left(f_{k}(s)\right)\right)\right. \\
&-g(s)] d s d u d v d w\}+\beta_{m} \gamma_{m}(t)-x(t) \mid \\
& \leq(1-\left.\alpha_{m}-\beta_{m}\right)\left|x_{m}(t)-x(t)\right| \\
&+\alpha_{m}\left|S_{L} x_{m}(t)-S_{L} x(t)\right|+\beta_{m}\left|\gamma_{m}(t)-x(t)\right| \\
& \leq\left(1-\alpha_{m}-\beta_{m}\right)\left|x_{m}(t)-x(t)\right| \\
&+\alpha_{m} \theta\left|x_{m}(t)-x(t)\right|+2 M \beta_{m}
\end{aligned}
$$

$$
\begin{aligned}
& \leq\left(1-(1-\theta) \alpha_{m}\right)\left|x_{m}(t)-x(t)\right|+2 M \beta_{m} \\
& \leq\left(1-(1-\theta) \alpha_{m}\right)\left\|x_{m}-x\right\|+2 M \beta_{m},
\end{aligned}
$$

which yielded that

$$
\begin{array}{r}
\left\|x_{m+1}-x\right\| \leq\left(1-(1-\theta) \alpha_{m}\right)\left\|x_{m}-x\right\|+2 M \beta_{m}, \\
m \in \mathbb{N}_{0} .
\end{array}
$$

That is, (22) holds. Thus Lemma 1, (23), and (24) ensure that $\lim _{m \rightarrow \infty} x_{m}=x$.

Next we show that (b) holds. Let $L_{1}, L_{2} \in\left(N+c_{1} M,(1-\right.$ $\left.c_{2}\right) M$ ) with $L_{1} \neq L_{2}$. As in the proof of (a), we conclude that for each $j \in\{1,2\}$ there exist $\theta_{j} \in(0,1), T_{j}>t_{0}+|a|+\tau+$ $\left|T_{0}\right|$, and $S_{L_{j}}: A(N, M) \rightarrow A(N, M)$ satisfying (25)-(27), where $\theta, T, S_{L}$ are replaced by $\theta_{j}, T_{j}$, and $S_{L_{j}}$, respectively, and the contraction mapping $S_{L_{j}}$ has a unique fixed point $z_{j} \in$ $A(N, M)$, which is also a bounded positive solution of (11). In order to prove (b), we need only to show that $z_{1} \neq z_{2}$. Put $T^{*}=\max \left\{T_{1}, T_{2}\right\}$. Note that for $t \geq T^{*}$ and $j \in\{1,2\}$

$$
\begin{aligned}
& z_{j}(t) \\
& =S_{L} z_{j}(t) \\
& =L_{j}-p(t) z_{j}(t-\tau) \\
& \quad+\int_{t}^{+\infty} \int_{w}^{+\infty} \int_{v}^{+\infty} \frac{1}{\alpha(u) \beta(v) \gamma(w)} \\
& \quad \times h\left(u, z_{j}\left(h_{1}(u)\right), \ldots, z_{j}\left(h_{k}(u)\right)\right) d u d v d w \\
& \quad-\int_{t}^{+\infty} \int_{w}^{+\infty} \int_{v}^{+\infty} \int_{u}^{+\infty} \frac{1}{\alpha(u) \beta(v) \gamma(w)} \\
& \quad \times\left[f\left(s, z_{j}\left(f_{1}(s)\right), \ldots, z_{j}\left(f_{k}(s)\right)\right)-g(s)\right] d s d u d v d w
\end{aligned}
$$

which together with (16) and (25) implies that for $t \geq T^{*}$

$$
\begin{aligned}
& \left|z_{1}(t)-z_{2}(t)\right| \\
& =\mid L_{1}-L_{2}-p(t)\left(z_{1}(t-\tau)-z_{2}(t-\tau)\right) \\
& \quad+\int_{t}^{+\infty} \int_{w}^{+\infty} \int_{v}^{+\infty} \frac{1}{\alpha(u) \beta(v) \gamma(w)} \\
& \quad \times\left[h\left(u, z_{1}\left(h_{1}(u)\right), \ldots, z_{1}\left(h_{k}(u)\right)\right)\right. \\
& \left.\quad-h\left(u, z_{2}\left(h_{1}(u)\right), \ldots, z_{2}\left(h_{k}(u)\right)\right)\right] d u d v d w \\
& \quad-\int_{t}^{+\infty} \int_{w}^{+\infty} \int_{v}^{+\infty} \int_{u}^{+\infty} \frac{1}{\alpha(u) \beta(v) \gamma(w)}
\end{aligned}
$$




$$
\begin{aligned}
\times & {\left[f\left(s, z_{1}\left(f_{1}(s)\right), \ldots, z_{1}\left(f_{k}(s)\right)\right)\right.} \\
& \left.-f\left(s, z_{2}\left(f_{1}(s)\right), \ldots, z_{2}\left(f_{k}(s)\right)\right)\right] d s d u d v d w \mid \\
\geq & \left|L_{1}-L_{2}\right|-\left(c_{1}+c_{2}\right)\left\|z_{1}-z_{2}\right\|-\left\|z_{1}-z_{2}\right\| \\
\times & \int_{T^{*}}^{+\infty} \int_{w}^{+\infty} \int_{v}^{+\infty} \frac{Q_{2}(u)}{|\alpha(u) \beta(v) \gamma(w)|} d u d v d w \\
- & \left\|z_{1}-z_{2}\right\| \\
\times & \int_{T^{*}}^{+\infty} \int_{w}^{+\infty} \int_{v}^{+\infty} \int_{u}^{+\infty} \frac{Q_{1}(s)}{|\alpha(u) \beta(v) \gamma(w)|} d s d u d v d w \\
\geq & \left|L_{1}-L_{2}\right|-\left\|z_{1}-z_{2}\right\| \max \left\{\theta_{1}, \theta_{2}\right\},
\end{aligned}
$$

which yields that

$$
\left\|z_{1}-z_{2}\right\| \geq \frac{\left|L_{1}-L_{2}\right|}{1+\max \left\{\theta_{1}, \theta_{2}\right\}}>0 .
$$

That is, $z_{1} \neq z_{2}$. This completes the proof.

Theorem 3. Assume that there exist constants $M, N, c_{1}, c_{2}$, and $T_{0} \geq t_{0}$ and functions $Q_{1}, Q_{2}, R_{1}$, and $R_{2} \in$ $C\left(\left[t_{0},+\infty\right), R^{+}\right)$satisfying $(16)-(18)$ and

$$
\begin{gathered}
0<\left(1-c_{2}\right) N<\left(1-c_{1}\right) M, \\
0 \leq c_{2} \leq p(t) \leq c_{1}<1, \quad t \geq T_{0} .
\end{gathered}
$$

Then

(a) for any $L \in\left(N+c_{1} M, M+c_{2} N\right)$, there exist $\theta \in(0,1)$ and $T>t_{0}+|a|+\tau+\left|T_{0}\right|$ such that, for each $x_{0} \in A(N, M)$, the Mann iterative sequence $\left\{x_{m}\right\}_{m \in \mathbb{N}_{0}}$ generated by the scheme (21) with (23) and (24) converges to a bounded positive solution $x \in$ $A(N, M)$ of (11) and has the error estimate (22);

(b) equation (11) has uncountably many bounded positive solutions in $A(N, M)$.

Proof. Let $L \in\left(N+c_{1} M, M+c_{2} N\right)$. It follows from (18) and (35) that there exist $\theta \in(0,1)$ and $T>t_{0}+|a|+\tau+\left|T_{0}\right|$ satisfying

$$
\begin{aligned}
\theta=c_{1} & +\int_{T}^{+\infty} \int_{w}^{+\infty} \int_{v}^{+\infty} \frac{Q_{2}(u)}{|\alpha(u) \beta(v) \gamma(w)|} d u d v d w \\
& +\int_{T}^{+\infty} \int_{w}^{+\infty} \int_{v}^{+\infty} \int_{u}^{+\infty} \frac{Q_{1}(s)}{|\alpha(u) \beta(v) \gamma(w)|} d s d u d v d w \\
& \int_{T}^{+\infty} \int_{w}^{+\infty} \int_{v}^{+\infty} \frac{R_{2}(u)}{|\alpha(u) \beta(v) \gamma(w)|} d u d v d w \\
& +\int_{T}^{+\infty} \int_{w}^{+\infty} \int_{v}^{+\infty} \int_{u}^{+\infty} \frac{R_{1}(s)+|g(s)|}{|\alpha(u) \beta(v) \gamma(w)|} d s d u d v d w \\
& <\min \left\{M+c_{2} N-L, L-N-c_{1} M\right\} .
\end{aligned}
$$

Define a mapping $S_{L}: A(N, M) \rightarrow C B([a,+\infty), \mathbb{R})$ by (27). Clearly $S_{L} x$ is continuous for each $x \in A(N, M)$. On account of (16), (17), (27), (35), (36), and (37), we infer that for $x, y \in$ $A(N, M)$ and $t \geq T$

$$
\begin{aligned}
& \left|S_{L} x(t)-S_{L} y(t)\right| \\
& \leq|p(t)||x(t-\tau)-y(t-\tau)| \\
& +\int_{t}^{+\infty} \int_{w}^{+\infty} \int_{v}^{+\infty} \frac{1}{|\alpha(u) \beta(v) \gamma(w)|} \\
& \times \mid h\left(u, x\left(h_{1}(u)\right), \ldots, x\left(h_{k}(u)\right)\right) \\
& -h\left(u, y\left(h_{1}(u)\right), \ldots, y\left(h_{k}(u)\right)\right) \mid d u d v d w \\
& +\int_{t}^{+\infty} \int_{w}^{+\infty} \int_{v}^{+\infty} \int_{u}^{+\infty} \frac{1}{|\alpha(u) \beta(v) \gamma(w)|} \\
& \times \mid f\left(s, x\left(f_{1}(s)\right), \ldots, x\left(f_{k}(s)\right)\right) \\
& -f\left(s, y\left(f_{1}(s)\right), \ldots, y\left(f_{k}(s)\right)\right) \mid d s d u d v d w \\
& \leq c_{1}\|x-y\|+\|x-y\| \\
& \times \int_{t}^{+\infty} \int_{w}^{+\infty} \int_{v}^{+\infty} \frac{Q_{2}(u)}{|\alpha(u) \beta(v) \gamma(w)|} d u d v d w \\
& +\|x-y\| \\
& \times \int_{t}^{+\infty} \int_{w}^{+\infty} \int_{v}^{+\infty} \int_{u}^{+\infty} \frac{Q_{1}(s)}{|\alpha(u) \beta(v) \gamma(w)|} d s d u d v d w \\
& \leq\left(c_{1}+\int_{T}^{+\infty} \int_{w}^{+\infty} \int_{v}^{+\infty} \frac{Q_{2}(u)}{|\alpha(u) \beta(v) \gamma(w)|} d u d v d w\right. \\
& \left.+\int_{T}^{+\infty} \int_{w}^{+\infty} \int_{v}^{+\infty} \int_{u}^{+\infty} \frac{Q_{1}(s)}{|\alpha(u) \beta(v) \gamma(w)|} d s d u d v d w\right) \\
& \times\|x-y\| \\
& =\theta\|x-y\| \text {, }
\end{aligned}
$$

$S_{L} x(t)$

$=L-p(t) x(t-\tau)$

$+\int_{t}^{+\infty} \int_{w}^{+\infty} \int_{v}^{+\infty} \frac{1}{\alpha(u) \beta(v) \gamma(w)}$

$\times h\left(u, x\left(h_{1}(u)\right), \ldots, x\left(h_{k}(u)\right)\right) d u d v d w$

$-\int_{t}^{+\infty} \int_{w}^{+\infty} \int_{v}^{+\infty} \int_{u}^{+\infty} \frac{1}{\alpha(u) \beta(v) \gamma(w)}$

$\times\left[f\left(s, x\left(f_{1}(s)\right), \ldots, x\left(f_{k}(s)\right)\right)-g(s)\right] d s d u d v d w$

$\leq L-c_{2} N$

$+\int_{t}^{+\infty} \int_{w}^{+\infty} \int_{v}^{+\infty} \frac{1}{|\alpha(u) \beta(v) \gamma(w)|}$

$\times\left|h\left(u, x\left(h_{1}(u)\right), \ldots, x\left(h_{k}(u)\right)\right)\right| d u d v d w$ 


$$
\begin{aligned}
& +\int_{t}^{+\infty} \int_{w}^{+\infty} \int_{v}^{+\infty} \int_{u}^{+\infty} \frac{1}{|\alpha(u) \beta(v) \gamma(w)|} \\
& \times\left[\left|f\left(s, x\left(f_{1}(s)\right), \ldots, x\left(f_{k}(s)\right)\right)\right|+|g(s)|\right] d s d u d v d w \\
\leq & L-c_{2} N \\
& +\int_{T}^{+\infty} \int_{w}^{+\infty} \int_{v}^{+\infty} \frac{R_{2}(u)}{|\alpha(u) \beta(v) \gamma(w)|} d u d v d w \\
& +\int_{T}^{+\infty} \int_{w}^{+\infty} \int_{v}^{+\infty} \int_{u}^{+\infty} \frac{R_{1}(s)+|g(s)|}{|\alpha(u) \beta(v) \gamma(w)|} d s d u d v d w \\
< & L-c_{2} N+\min \left\{M+c_{2} N-L, L-N-c_{1} M\right\} \\
\leq & M, \\
S_{L} x & (t) \\
\geq & L-c_{1} M \\
& -\int_{t}^{+\infty} \int_{w}^{+\infty} \int_{v}^{+\infty} \frac{1}{|\alpha(u) \beta(v) \gamma(w)|} \\
& \times\left|h\left(u, x\left(h_{1}(u)\right), \ldots, x\left(h_{k}(u)\right)\right)\right| d u d v d w \\
> & L-c_{1} M-\min \left\{M+c_{2} N-L, L-N-c_{1} M\right\} \\
\geq & N, \\
& -\int_{t}^{+\infty} \int_{w}^{+\infty} \int_{v}^{+\infty} \int_{u}^{+\infty} \frac{1}{|\alpha(u) \beta(v) \gamma(w)|} \\
& \times\left|\left[f\left(s, x\left(f_{1}(s)\right), \ldots, x\left(f_{k}(s)\right)\right)-g(s)\right]\right| d s d u d v d w \\
\geq & L-c_{1} M \\
& -\int_{T}^{+\infty} \int_{w}^{+\infty} \int_{v}^{+\infty} \frac{R_{2}(u)}{|\alpha(u) \beta(v) \gamma(w)|} d u d v d w \\
& \int_{v}^{+\infty} \int_{u}^{+\infty} \frac{R_{1}(s)+|g(s)|}{|\alpha(u) \beta(v) \gamma(w)|} d s d u d v d w \\
&
\end{aligned}
$$

which imply that (29) holds. The rest of the proof is similar to that of Theorem 2 and is omitted. This completes the proof.

Theorem 4. Assume that there exist constants $M, N, c_{1}, c_{2}$ and $T_{0} \geq t_{0}$ and functions $Q_{1}, Q_{2}, R_{1}$ and $R_{2} \in C\left(\left[t_{0},+\infty\right), \mathbb{R}^{+}\right)$ satisfying (17), (18), and

$$
\begin{gathered}
0<\left(1-c_{2}\right) N<\left(1-c_{1}\right) M ; \\
-1<-c_{1} \leq p(t) \leq-c_{2} \leq 0, \quad t \geq T_{0} .
\end{gathered}
$$

Then

(a) for any $L \in\left(\left(1-c_{2}\right) N,\left(1-c_{1}\right) M\right)$, there exist $\theta \in(0,1)$ and $T>t_{0}+|a|+\tau+\left|T_{0}\right|$ such that, for each $x_{0} \in A(N, M)$, the Mann iterative sequence $\left\{x_{m}\right\}_{m \in \mathbb{N}_{0}}$ generated by the scheme (21) with (23) and (24) converges to a bounded positive solution $x \in$ $A(N, M)$ of (11) and has the error estimate (22); (b) equation (11) has uncountably many bounded positive solutions in $A(N, M)$.

Proof. Let $L \in\left(\left(1-c_{2}\right) N,\left(1-c_{1}\right) M\right)$. Equations (18) and (39) guarantee that there exist $\theta \in(0,1)$ and $T>t_{0}+|a|+\tau+\left|T_{0}\right|$ satisfying (36) and

$$
\begin{aligned}
& \int_{T}^{+\infty} \int_{w}^{+\infty} \int_{v}^{+\infty} \frac{R_{2}(u)}{|\alpha(u) \beta(v) \gamma(w)|} d u d v d w \\
& \quad+\int_{T}^{+\infty} \int_{w}^{+\infty} \int_{v}^{+\infty} \int_{u}^{+\infty} \frac{R_{1}(s)+|g(s)|}{|\alpha(u) \beta(v) \gamma(w)|} d s d u d v d w \\
& <\min \left\{\left(1-c_{1}\right) M-L,\left(c_{2}-1\right) N+L\right\} .
\end{aligned}
$$

Let the mapping $S_{L}: A(N, M) \rightarrow C B([a,+\infty), \mathbb{R})$ be defined by (27). Using (17), (27), (39), and (40), we deduce that for any $x \in A(N, M)$ and $t \geq T$

$$
\begin{aligned}
& S_{L} x(t) \\
&=L-p(t) x(t-\tau) \\
& \quad+\int_{t}^{+\infty} \int_{w}^{+\infty} \int_{v}^{+\infty} \frac{1}{\alpha(u) \beta(v) \gamma(w)} \\
& \quad \times h\left(u, x\left(h_{1}(u)\right), \ldots, x\left(h_{k}(u)\right)\right) d u d v d w \\
& \quad-\int_{t}^{+\infty} \int_{w}^{+\infty} \int_{v}^{+\infty} \int_{u}^{+\infty} \frac{1}{\alpha(u) \beta(v) \gamma(w)} \\
& \quad \times\left[f\left(s, x\left(f_{1}(s)\right), \ldots, x\left(f_{k}(s)\right)\right)-g(s)\right] d s d u d v d w \\
& \leq L+c_{1} M \\
&+\int_{t}^{+\infty} \int_{w}^{+\infty} \int_{v}^{+\infty} \frac{1}{|\alpha(u) \beta(v) \gamma(w)|} \\
& \times\left|h\left(u, x\left(h_{1}(u)\right), \ldots, x\left(h_{k}(u)\right)\right)\right| d u d v d w \\
&+\int_{t}^{+\infty} \int_{w}^{+\infty} \int_{v}^{+\infty} \int_{u}^{+\infty} \frac{1}{|\alpha(u) \beta(v) \gamma(w)|} \\
& \times\left[\left|f\left(s, x\left(f_{1}(s)\right), \ldots, x\left(f_{k}(s)\right)\right)\right|\right. \\
&+|g(s)|] d s d u d v d w \\
& \leq+c_{1} M \\
&+\int_{T}^{+\infty} \int_{w}^{+\infty} \int_{v}^{+\infty} \frac{R_{2}(u)}{|\alpha(u) \beta(v) \gamma(w)|} d u d v d w \\
& \int_{w}^{+\infty} \int_{v}^{+\infty} \int_{u}^{+\infty} \frac{R_{1}(s)+|g(s)|}{|\alpha(u) \beta(v) \gamma(w)|} d s d u d v d w
\end{aligned}
$$




$$
\begin{aligned}
& <L+c_{1} M+\min \left\{\left(1-c_{1}\right) M-L,\left(c_{2}-1\right) N+L\right\} \\
& \leq M \text {, } \\
& S_{L} x(t) \\
& \geq L+c_{2} N \\
& -\int_{T}^{+\infty} \int_{w}^{+\infty} \int_{v}^{+\infty} \frac{R_{2}(u)}{|\alpha(u) \beta(v) \gamma(w)|} d u d v d w \\
& -\int_{T}^{+\infty} \int_{w}^{+\infty} \int_{v}^{+\infty} \int_{u}^{+\infty} \frac{R_{1}(s)+|g(s)|}{|\alpha(u) \beta(v) \gamma(w)|} d s d u d v d w \\
& >L+c_{2} N-\min \left\{\left(1-c_{1}\right) M-L,\left(c_{2}-1\right) N+L\right\} \\
& \geq N
\end{aligned}
$$

which mean that (29) holds. That is, $S_{L}$ is a contraction mapping and possesses a unique fixed point $x \in A(N, M)$, which is a bounded positive solution of (11). The rest of the proof is similar to that of Theorem 2 and is omitted. This completes the proof.

Theorem 5. Assume that there exist constants $M, N$, and $T_{0} \geq t_{0}$ and functions $Q_{1}, Q_{2}, R_{1}$, and $R_{2} \in C\left(\left[t_{0},+\infty\right), \mathbb{R}^{+}\right)$ satisfying (16)-(18) and

$$
0<N<M, \quad p(t)=1, \quad t \geq T_{0} .
$$

Then

(a) for any $L \in(N, M)$, there exist $\theta \in(0,1)$ and $T>$ $t_{0}+|a|+\tau+\left|T_{0}\right|$ such that, for each $x_{0} \in A(N, M)$, the Mann iterative sequence $\left\{x_{m}\right\}_{m \in \mathbb{N}_{0}}$ generated by the scheme

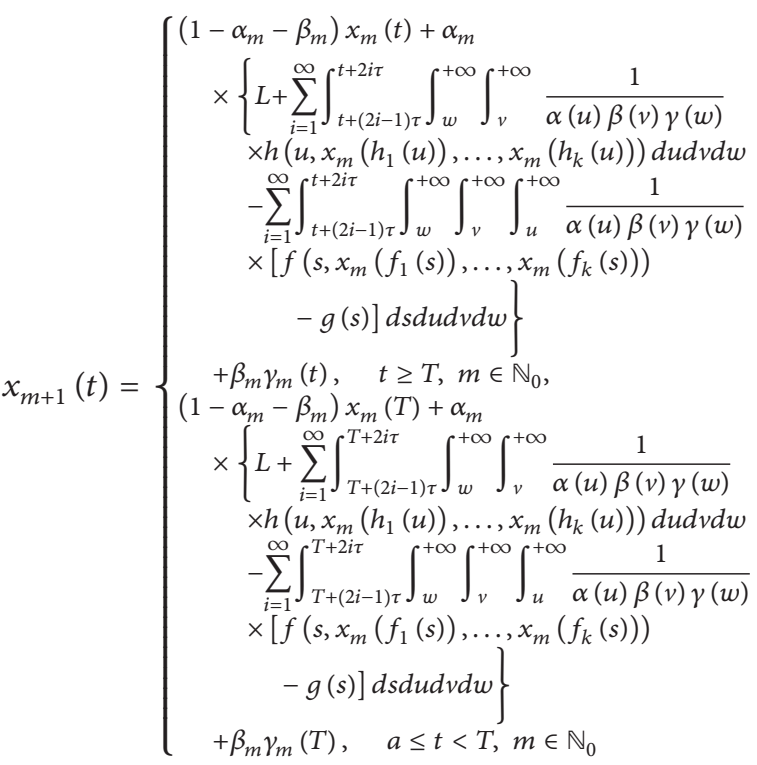

converges to a bounded positive solution $x \in A(N, M)$ of (11) and has the error estimate (22), where $\left\{\gamma_{m}\right\}_{m \in \mathbb{N}_{0}}$ is an arbitrary sequence in $A(N, M)$ and $\left\{\alpha_{m}\right\}_{m \in \mathbb{N}_{0}}$ and $\left\{\beta_{m}\right\}_{m \in \mathbb{N}_{0}}$ are any sequences in $[0,1]$ satisfying $(23)$ and (24); (b) equation (11) possesses uncountably many bounded positive solutions in $A(N, M)$.

Proof. First of all, we show that (a) holds. Let $L \in(N, M)$. It follows from (18) that there exist $\theta \in(0,1)$ and $T>t_{0}+|a|+$ $\tau+\left|T_{0}\right|$ satisfying

$$
\begin{aligned}
\theta= & \int_{T}^{+\infty} \int_{w}^{+\infty} \int_{v}^{+\infty} \frac{Q_{2}(u)}{|\alpha(u) \beta(v) \gamma(w)|} d u d v d w \\
& +\int_{T}^{+\infty} \int_{w}^{+\infty} \int_{v}^{+\infty} \int_{u}^{+\infty} \frac{Q_{1}(s)}{|\alpha(u) \beta(v) \gamma(w)|} d s d u d v d w
\end{aligned}
$$

$$
\begin{aligned}
& \int_{T}^{+\infty} \int_{w}^{+\infty} \int_{v}^{+\infty} \frac{R_{2}(u)}{|\alpha(u) \beta(v) \gamma(w)|} d u d v d w \\
& \quad+\int_{T}^{+\infty} \int_{w}^{+\infty} \int_{v}^{+\infty} \int_{u}^{+\infty} \frac{R_{1}(s)+|g(s)|}{|\alpha(u) \beta(v) \gamma(w)|} d s d u d v d w \\
& \quad<\min \{M-L, L-N\} .
\end{aligned}
$$

Define a mapping $S_{L}: A(N, M) \rightarrow C B([a,+\infty), \mathbb{R})$ by

$$
S_{L} x(t)=\left\{\begin{array}{c}
L+\sum_{i=1}^{\infty} \int_{t+(2 i-1) \tau}^{t+2 i \tau} \int_{w}^{+\infty} \int_{v}^{+\infty} \frac{1}{\alpha(u) \beta(v) \gamma(w)} \\
\quad \times h\left(u, x\left(h_{1}(u)\right), \ldots, x\left(h_{k}(u)\right)\right) d u d v d w \\
-\sum_{i=1}^{\infty} \int_{t+(2 i-1) \tau}^{t+2 i \tau} \int_{w}^{+\infty} \int_{v}^{+\infty} \int_{u}^{+\infty} \frac{1}{\alpha(u) \beta(v) \gamma(w)} \\
\quad \times\left[f\left(s, x\left(f_{1}(s)\right), \ldots, x\left(f_{k}(s)\right)\right)\right. \\
-g(s)] d s d u d v d w, \\
t \geq T, \quad x \in A(N, M), \\
S_{L} x(T), \quad a \leq t<T, \quad x \in A(N, M) .
\end{array}\right.
$$

Clearly, $S_{L} x$ is continuous for each $x \in A(N, M)$. Notice that (16), (17), (42), and (44)-(46) ensure that for $x, y \in A(N, M)$ and $t \geq T$

$$
\begin{aligned}
& \left|S_{L} x(t)-S_{L} y(t)\right| \\
& \leq \sum_{i=1}^{\infty} \int_{t+(2 i-1) \tau}^{t+2 i \tau} \int_{w}^{+\infty} \int_{v}^{+\infty} \frac{1}{|\alpha(u) \beta(v) \gamma(w)|} \\
& \quad \times \mid h\left(u, x\left(h_{1}(u)\right), \ldots, x\left(h_{k}(u)\right)\right) \\
& \quad-h\left(u, y\left(h_{1}(u)\right), \ldots, y\left(h_{k}(u)\right)\right) \mid d u d v d w \\
& \quad+\sum_{i=1}^{\infty} \int_{t+(2 i-1) \tau}^{t+2 i \tau} \int_{w}^{+\infty} \int_{v}^{+\infty} \int_{u}^{+\infty} \frac{1}{|\alpha(u) \beta(v) \gamma(w)|}
\end{aligned}
$$




$$
\begin{aligned}
\times & \mid f\left(s, x\left(f_{1}(s)\right), \ldots, x\left(f_{k}(s)\right)\right) \\
& -f\left(s, y\left(f_{1}(s)\right), \ldots, y\left(f_{k}(s)\right)\right) \mid d s d u d v d w 2 \leq\|x-y\| \\
\times & \sum_{i=1}^{\infty} \int_{t+(2 i-1) \tau}^{t+2 i \tau} \int_{w}^{+\infty} \int_{v}^{+\infty} \frac{Q_{2}(u)}{|\alpha(u) \beta(v) \gamma(w)|} d u d v d w \\
+ & \|x-y\| \\
\times & \sum_{i=1}^{\infty} \int_{t+(2 i-1) \tau}^{t+2 i \tau} \int_{w}^{+\infty} \int_{v}^{+\infty} \int_{u}^{+\infty} \frac{Q_{1}(s)}{|\alpha(u) \beta(v) \gamma(w)|} d s d u d v d w \\
\leq & \left(\int_{T}^{+\infty} \int_{w}^{+\infty} \int_{v}^{+\infty} \frac{Q_{2}(u)}{|\alpha(u) \beta(v) \gamma(w)|} d u d v d w\right. \\
& \left.+\int_{T}^{+\infty} \int_{w}^{+\infty} \int_{v}^{+\infty} \int_{u}^{+\infty} \frac{Q_{1}(s)}{|\alpha(u) \beta(v) \gamma(w)|} d s d u d v d w\right)
\end{aligned}
$$$$
\times\|x-y\|
$$$$
=\theta\|x-y\|,
$$$$
S_{L} x(t)
$$$$
=L+\sum_{i=1}^{\infty} \int_{t+(2 i-1) \tau}^{t+2 i \tau} \int_{w}^{+\infty} \int_{v}^{+\infty} \frac{1}{\alpha(u) \beta(v) \gamma(w)}
$$$$
\times h\left(u, x\left(h_{1}(u)\right), \ldots, x\left(h_{k}(u)\right)\right) d u d v d w
$$$$
-\sum_{i=1}^{\infty} \int_{t+(2 i-1) \tau}^{t+2 i \tau} \int_{w}^{+\infty} \int_{v}^{+\infty} \int_{u}^{+\infty} \frac{1}{\alpha(u) \beta(v) \gamma(w)}
$$$$
\times\left[f\left(s, x\left(f_{1}(s)\right), \ldots, x\left(f_{k}(s)\right)\right)-g(s)\right] d s d u d v d w
$$$$
\leq L+\int_{t}^{+\infty} \int_{w}^{+\infty} \int_{v}^{+\infty} \frac{R_{2}(u)}{|\alpha(u) \beta(v) \gamma(w)|} d u d v d w
$$$$
+\int_{t}^{+\infty} \int_{w}^{+\infty} \int_{v}^{+\infty} \int_{u}^{+\infty} \frac{R_{1}(s)+|g(s)|}{|\alpha(u) \beta(v) \gamma(w)|} d s d u d v d w
$$$$
<L+\min \{M-L, L-N\}
$$$$
\leq M \text {, }
$$$$
S_{L} x(t)
$$$$
\geq L-\int_{t}^{+\infty} \int_{w}^{+\infty} \int_{v}^{+\infty} \frac{R_{2}(u)}{|\alpha(u) \beta(v) \gamma(w)|} d u d v d w
$$$$
-\int_{t}^{+\infty} \int_{w}^{+\infty} \int_{v}^{+\infty} \int_{u}^{+\infty} \frac{R_{1}(s)+|g(s)|}{|\alpha(u) \beta(v) \gamma(w)|} d s d u d v d w
$$$$
>L-\min \{M-L, L-N\}
$$$$
\geq N \text {, }
$$

which imply that (29) holds. That is, $S_{L}$ is a contraction mapping and has a unique fixed point $x \in A(N, M)$. It follows that

$$
\begin{aligned}
x(t) & L+\sum_{i=1}^{\infty} \int_{t+(2 i-1) \tau}^{t+2 i \tau} \int_{w}^{+\infty} \int_{v}^{+\infty} \frac{1}{\alpha(u) \beta(v) \gamma(w)} \\
& \times h\left(u, x\left(h_{1}(u)\right), \ldots, x\left(h_{k}(u)\right)\right) d u d v d w \\
& -\sum_{i=1}^{\infty} \int_{t+(2 i-1) \tau}^{t+2 i \tau} \int_{w}^{+\infty} \int_{v}^{+\infty} \int_{u}^{+\infty} \frac{1}{\alpha(u) \beta(v) \gamma(w)} \\
& \times\left[f\left(s, x\left(f_{1}(s)\right), \ldots, x\left(f_{k}(s)\right)\right)\right. \\
x & (t-\tau) \\
= & L+\sum_{i=1}^{\infty} \int_{t+(2 i-2) \tau}^{t+(2 i-1) \tau} \int_{w}^{+\infty} \int_{v}^{+\infty} \frac{1}{\alpha(u) \beta(v) \gamma(w)} \\
& \times h\left(u, x\left(h_{1}(u)\right), \ldots, x\left(h_{k}(u)\right)\right) d u d v d w \\
& -\sum_{i=1}^{\infty} \int_{t+(2 i-2) \tau}^{t+(2 i-1) \tau} \int_{w}^{+\infty} \int_{v}^{+\infty} \int_{u}^{+\infty} \frac{1}{\alpha(u) \beta(v) \gamma(w)} \\
& \times\left[f\left(s, x\left(f_{1}(s)\right), \ldots, x\left(f_{k}(s)\right)\right)\right. \\
& -g(s)] d s d u d v d w, t \geq T+\tau .
\end{aligned}
$$

Adding (48) and (49), we infer that

$$
\begin{aligned}
x( & (t)+x(t-\tau) \\
= & 2 L+\sum_{i=1}^{\infty}\left[\int_{t+(2 i-1) \tau}^{t+2 i \tau}+\int_{t+(2 i-2) \tau}^{t+(2 i-1) \tau}\right] \\
& \times \int_{w}^{+\infty} \int_{v}^{+\infty} \frac{1}{\alpha(u) \beta(v) \gamma(w)} \\
& \times h\left(u, x\left(h_{1}(u)\right), \ldots, x\left(h_{k}(u)\right)\right) d u d v d w \\
& -\sum_{i=1}^{\infty}\left[\int_{t+(2 i-1) \tau}^{t+2 i \tau}+\int_{t+(2 i-2) \tau}^{t+(2 i-1) \tau}\right] \\
& \times \int_{w}^{+\infty} \int_{v}^{+\infty} \int_{u}^{+\infty} \frac{1}{\alpha(u) \beta(v) \gamma(w)} \\
& \times\left[f\left(s, x\left(f_{1}(s)\right), \ldots, x\left(f_{k}(s)\right)\right)-g(s)\right] d s d u d v d w \\
= & 2 L+\int_{t}^{+\infty} \int_{w}^{+\infty} \int_{v}^{+\infty} \frac{1}{\alpha(u) \beta(v) \gamma(w)} \\
& \times h\left(u, x\left(h_{1}(u)\right), \ldots, x\left(h_{k}(u)\right)\right) d u d v d w
\end{aligned}
$$




$$
\begin{gathered}
-\int_{t}^{+\infty} \int_{w}^{+\infty} \int_{v}^{+\infty} \int_{u}^{+\infty} \frac{1}{\alpha(u) \beta(v) \gamma(w)} \\
\times\left[f\left(s, x\left(f_{1}(s)\right), \ldots, x\left(f_{k}(s)\right)\right)\right. \\
\quad-g(s)] d s d u d v d w, \quad t \geq T+\tau
\end{gathered}
$$

which yields that $x$ is a bounded positive solution of (11). By means of (29), (43), and (46), we know that for any $t \geq T$ and $m \in \mathbb{N}_{0}$

$$
\begin{aligned}
& \left|x_{m+1}(t)-x(t)\right| \\
& =\mid\left(1-\alpha_{m}-\beta_{m}\right) x_{m}(t)+\alpha_{m} \\
& \quad \times\left\{L+\sum_{i=1}^{\infty} \int_{t+(2 i-1) \tau}^{t+2 i \tau} \int_{w}^{+\infty} \int_{v}^{+\infty} \frac{1}{\alpha(u) \beta(v) \gamma(w)}\right. \\
& \quad \times h\left(u, x_{m}\left(h_{1}(u)\right), \ldots, x_{m}\left(h_{k}(u)\right)\right) d u d v d w \\
& \quad-\sum_{i=1}^{\infty} \int_{t+(2 i-1) \tau}^{t+2 i \tau} \int_{v}^{+\infty} \int_{u}^{+\infty} \frac{1}{\alpha(u) \beta(v) \gamma(w)} \\
& \quad \times\left[f\left(s, x_{m}\left(f_{1}(s)\right), \ldots, x_{m}\left(f_{k}(s)\right)\right)\right. \\
& \quad-g(s)] d s d u d v d w\}+\beta_{m} \gamma_{m}(t)-x(t) \mid \\
& \leq\left(1-\alpha_{m}-\beta_{m}\right)\left|x_{m}(t)-x(t)\right| \\
& +\alpha_{m}\left|S_{L} x_{m}(t)-S_{L} x(t)\right|+\beta_{m}\left|\gamma_{m}(t)-x(t)\right| \\
& \leq\left(1-\alpha_{m}-\beta_{m}\right)\left|x_{m}(t)-x(t)\right| \\
& +\alpha_{m} \theta\left|x_{m}(t)-x(t)\right|+2 M \beta_{m} \\
& \left.\leq(1-\theta) \alpha_{m}\right)\left|x_{m}(t)-x(t)\right|+2 M \beta_{m},
\end{aligned}
$$

which gives (22). Thus Lemma 1, (23), and (24) ensure that $\lim _{m \rightarrow \infty} x_{m}=x$.

Now we show that (b) holds. Let $L_{1}, L_{2} \in(N, M)$ with $L_{1} \neq L_{2}$. As in the proof of (a), for each $\in\{1,2\}$, we infer that there exists $\theta_{j} \in(0,1), T_{j}>t_{0}+|a|+\tau+\left|T_{0}\right|$, and $S_{L_{j}}$ : $A(N, M) \rightarrow A(N, M)$ satisfying (44)-(46), where $\theta, T, S_{L}$ are replaced by $\theta_{j}, T_{j}, S_{L_{j}}$, respectively, and the contraction mapping $S_{L_{j}}$ possesses a unique fixed point $z_{j} \in A(N, M)$, and $z_{j}$ is a bounded positive solution of (11); that is,

$$
\begin{aligned}
& z_{j}(t) \\
& =S_{L} z_{j}(t) \\
& =L_{j}+\sum_{i=1}^{\infty} \int_{t+(2 i-1) \tau}^{t+2 i \tau} \int_{w}^{+\infty} \int_{v}^{+\infty} \frac{1}{\alpha(u) \beta(v) \gamma(w)} \\
& \quad \times h\left(u, z_{j}\left(h_{1}(u)\right), \ldots, z_{j}\left(h_{k}(u)\right)\right) d u d v d w
\end{aligned}
$$

$$
\begin{aligned}
& -\sum_{i=1}^{\infty} \int_{t+(2 i-1) \tau}^{t+2 i \tau} \int_{w}^{+\infty} \int_{v}^{+\infty} \int_{u}^{+\infty} \frac{1}{\alpha(u) \beta(v) \gamma(w)} \\
& \times\left[f\left(s, z_{j}\left(f_{1}(s)\right), \ldots, z_{j}\left(f_{k}(s)\right)\right)\right. \\
& -g(s)] d s d u d v d w, \quad t \geq T_{j} .
\end{aligned}
$$

Put $T^{*}=\max \left\{T_{1}, T_{2}\right\}$. Using (16), (44), and (52), we conclude that for $t>T^{*}$

$$
\begin{aligned}
& \left|z_{1}(t)-z_{2}(t)\right| \\
& =\mid L_{1}-L_{2} \\
& +\sum_{i=1}^{\infty} \int_{t+(2 i-1) \tau}^{t+2 i \tau} \int_{w}^{+\infty} \int_{v}^{+\infty} \frac{1}{\alpha(u) \beta(v) \gamma(w)} \\
& \times\left[h\left(u, z_{1}\left(h_{1}(u)\right), \ldots, z_{1}\left(h_{k}(u)\right)\right)\right. \\
& \left.-h\left(u, z_{2}\left(h_{1}(u)\right), \ldots, z_{2}\left(h_{k}(u)\right)\right)\right] d u d v d w \\
& -\sum_{i=1}^{\infty} \int_{t+(2 i-1) \tau}^{t+2 i \tau} \int_{w}^{+\infty} \int_{v}^{+\infty} \int_{u}^{+\infty} \frac{1}{\alpha(u) \beta(v) \gamma(w)} \\
& \times\left[f\left(s, z_{1}\left(f_{1}(s)\right), \ldots, z_{1}\left(f_{k}(s)\right)\right)\right. \\
& \left.-f\left(s, z_{2}\left(f_{1}(s)\right), \ldots, z_{2}\left(f_{k}(s)\right)\right)\right] d s d u d v d w \mid \\
& \geq\left|L_{1}-L_{2}\right|-\left\|z_{1}-z_{2}\right\| \\
& \times \sum_{i=1}^{\infty} \int_{t+(2 i-1) \tau}^{t+2 i \tau} \int_{w}^{+\infty} \int_{v}^{+\infty} \frac{Q_{2}(u)}{|\alpha(u) \beta(v) \gamma(w)|} d u d v d w \\
& -\left\|z_{1}-z_{2}\right\| \\
& \times \sum_{i=1}^{\infty} \int_{t+(2 i-1) \tau}^{t+2 i \tau} \int_{w}^{+\infty} \int_{v}^{+\infty} \int_{u}^{+\infty} \frac{Q_{1}(s)}{|\alpha(u) \beta(v) \gamma(w)|} d s d u d v d w \\
& \geq\left|L_{1}-L_{2}\right|-\left\|z_{1}-z_{2}\right\| \\
& \times\left(\int_{T^{*}}^{+\infty} \int_{w}^{+\infty} \int_{v}^{+\infty} \frac{Q_{2}(u)}{|\alpha(u) \beta(v) \gamma(w)|} d u d v d w\right. \\
& \left.+\int_{T^{*}}^{+\infty} \int_{w}^{+\infty} \int_{v}^{+\infty} \int_{u}^{+\infty} \frac{Q_{1}(s)}{|\alpha(u) \beta(v) \gamma(w)|} d s d u d v d w\right) \\
& \geq\left|L_{1}-L_{2}\right|-\left\|z_{1}-z_{2}\right\| \max \left\{\theta_{1}, \theta_{2}\right\},
\end{aligned}
$$

which yields that

$$
\left\|z_{1}-z_{2}\right\| \geq \frac{\left|L_{1}-L_{2}\right|}{1+\max \left\{\theta_{1}, \theta_{2}\right\}}>0 ;
$$

that is, $z_{1} \neq z_{2}$. This completes the proof. 
Theorem 6. Assume that there exist constants $M, N$ and $T_{0} \geq t_{0}$ and functions $Q_{1}, Q_{2}, R_{1}$ and $R_{2} \in C\left(\left[t_{0},+\infty\right), \mathbb{R}^{+}\right)$ satisfying (16), (17), and

$$
\begin{gathered}
0<N<M, \quad p(t)=-1, \quad t \geq T_{0}, \\
\sum_{i=1}^{\infty} \int_{t_{0}+i \tau}^{+\infty} \int_{w}^{+\infty} \int_{v}^{+\infty} \frac{\max \left\{Q_{2}(u), R_{2}(u)\right\}}{|\alpha(u) \beta(v) \gamma(w)|} d u d v d w<+\infty \\
\sum_{i=1}^{\infty} \int_{t_{0}+i \tau}^{+\infty} \int_{w}^{+\infty} \int_{v}^{+\infty} \int_{u}^{+\infty} \frac{\max \left\{Q_{1}(s), R_{1}(s),|g(s)|\right\}}{|\alpha(u) \beta(v) \gamma(w)|}<+\infty .
\end{gathered}
$$

Then

(a) for any $L \in(N, M)$, there exist $\theta \in(0,1)$ and $T>$ $t_{0}+|a|+\tau+\left|T_{0}\right|$ such that, for each $x_{0} \in A(N, M)$, the Mann iterative sequence $\left\{x_{m}\right\}_{m \in \mathbb{N}_{0}}$ generated by the scheme

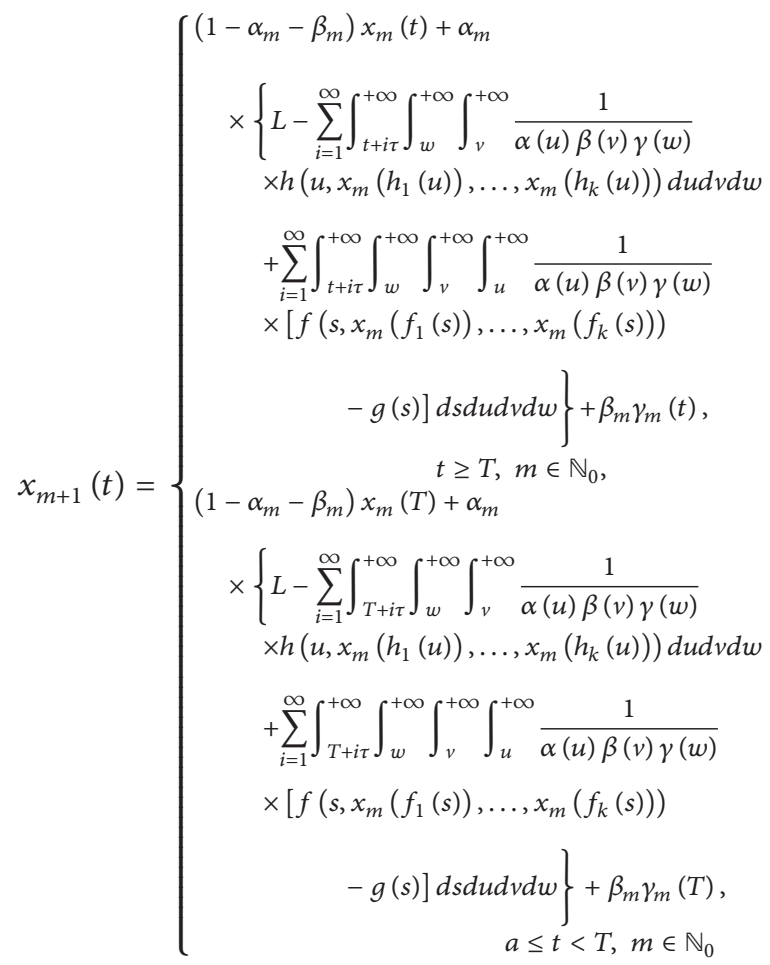

converges to a bounded positive solution $x \in A(N, M)$ of (11) and has the error estimate (22), where $\left\{\gamma_{m}\right\}_{m \in \mathbb{N}_{0}}$ is an arbitrary sequence in $A(N, M)$, and $\left\{\alpha_{m}\right\}_{m \in \mathbb{N}_{0}}$ and $\left\{\beta_{m}\right\}_{m \in \mathbb{N}_{0}}$ are any sequences in $[0,1]$ satisfying $(23)$ and (24);

(b) equation (11) possesses uncountably many bounded positive solutions in $A(N, M)$.
Proof. Firstly, we show that (a) holds. Let $L \in(N, M)$. It follows from (56) that there exist $\theta \in(0,1)$ and $T>t_{0}+$ $|a|+\tau+\left|T_{0}\right|$ satisfying

$$
\begin{aligned}
\theta= & \sum_{i=1}^{\infty} \int_{T+i \tau}^{+\infty} \int_{w}^{+\infty} \int_{v}^{+\infty} \frac{Q_{2}(u)}{|\alpha(u) \beta(v) \gamma(w)|} d u d v d w \\
& +\sum_{i=1}^{\infty} \int_{T+i \tau}^{+\infty} \int_{w}^{+\infty} \int_{v}^{+\infty} \int_{u}^{+\infty} \frac{Q_{1}(s)}{|\alpha(u) \beta(v) \gamma(w)|} d s d u d v d w,
\end{aligned}
$$

$$
\begin{aligned}
& \sum_{i=1}^{\infty} \int_{T+i \tau}^{+\infty} \int_{w}^{+\infty} \int_{v}^{+\infty} \frac{R_{2}(u)}{|\alpha(u) \beta(v) \gamma(w)|} d u d v d w \\
& \quad+\sum_{i=1}^{\infty} \int_{T+i \tau}^{+\infty} \int_{w}^{+\infty} \int_{v}^{+\infty} \int_{u}^{+\infty} \frac{R_{1}(s)+|g(s)|}{|\alpha(u) \beta(v) \gamma(w)|} d s d u d v d w
\end{aligned}
$$$$
<\min \{M-L, L-N\} \text {. }
$$

Define a mapping $S_{L}: A(N, M) \rightarrow C B([a,+\infty), \mathbb{R})$ by

$$
S_{L} x(t)=\left\{\begin{array}{c}
L-\sum_{i=1}^{\infty} \int_{t+i \tau}^{+\infty} \int_{w}^{+\infty} \int_{v}^{+\infty} \frac{1}{\alpha(u) \beta(v) \gamma(w)} \\
\times h\left(u, x\left(h_{1}(u)\right), \ldots, x\left(h_{k}(u)\right)\right) d u d v d w \\
+\sum_{i=1}^{\infty} \int_{t+i \tau}^{+\infty} \int_{w}^{+\infty} \int_{v}^{+\infty} \int_{u}^{+\infty} \frac{1}{\alpha(u) \beta(v) \gamma(w)} \\
\times\left[f\left(s, x\left(f_{1}(s)\right), \ldots, x\left(f_{k}(s)\right)\right)\right. \\
-g(s)] d s d u d v d w, \\
t \geq T, \quad x \in A(N, M), \\
S_{L} x(T), \quad a \leq t<T, x \in A(N, M) .
\end{array}\right.
$$

Clearly, $S_{L} x$ is continuous for each $x \in A(N, M)$. In light of (16), (17), and (58)-(60), we conclude that for $x, y \in A(N, M)$ and $t \geq T$

$$
\begin{aligned}
& \left|S_{L} x(t)-S_{L} y(t)\right| \\
& \leq \sum_{i=1}^{\infty} \int_{t+i \tau}^{+\infty} \int_{w}^{+\infty} \int_{v}^{+\infty} \frac{1}{|\alpha(u) \beta(v) \gamma(w)|} \\
& \quad \times \mid h\left(u, x\left(h_{1}(u)\right), \ldots, x\left(h_{k}(u)\right)\right) \\
& \quad-h\left(u, y\left(h_{1}(u)\right), \ldots, y\left(h_{k}(u)\right)\right) \mid d u d v d w \\
& \quad+\sum_{i=1}^{\infty} \int_{t+i \tau}^{+\infty} \int_{w}^{+\infty} \int_{v}^{+\infty} \int_{u}^{+\infty} \frac{1}{|\alpha(u) \beta(v) \gamma(w)|} \\
& \quad-f\left(s, x\left(f_{1}(s)\right), \ldots, x\left(f_{k}(s)\right)\right)
\end{aligned}
$$




$$
\begin{aligned}
\leq & \|x-y\| \\
& \times \sum_{i=1}^{\infty} \int_{t+i \tau}^{+\infty} \int_{w}^{+\infty} \int_{v}^{+\infty} \frac{Q_{2}(u)}{|\alpha(u) \beta(v) \gamma(w)|} d u d v d w \\
& +\|x-y\| \\
& \times \sum_{i=1}^{\infty} \int_{t+i \tau}^{+\infty} \int_{w}^{+\infty} \int_{v}^{+\infty} \int_{u}^{+\infty} \frac{Q_{1}(s)}{|\alpha(u) \beta(v) \gamma(w)|} d s d u d v d w \\
\leq & \left(\sum_{i=1}^{\infty} \int_{T+i \tau}^{+\infty} \int_{w}^{+\infty} \int_{v}^{+\infty} \frac{Q_{2}(u)}{|\alpha(u) \beta(v) \gamma(w)|} d u d v d w\right. \\
& \left.+\sum_{i=1}^{\infty} \int_{T+i \tau}^{+\infty} \int_{w}^{+\infty} \int_{v}^{+\infty} \int_{u}^{+\infty} \frac{Q_{1}(s)}{|\alpha(u) \beta(v) \gamma(w)|} d s d u d v d w\right) \\
& \times\|x-y\| \\
= & \theta\|x-y\|,
\end{aligned}
$$$$
S_{L} x(t)
$$$$
=L-\sum_{i=1}^{\infty} \int_{t+i \tau}^{+\infty} \int_{w}^{+\infty} \int_{v}^{+\infty} \frac{1}{\alpha(u) \beta(v) \gamma(w)}
$$$$
\times h\left(u, x\left(h_{1}(u)\right), \ldots, x\left(h_{k}(u)\right)\right) d u d v d w
$$$$
+\sum_{i=1}^{\infty} \int_{t+i \tau}^{+\infty} \int_{w}^{+\infty} \int_{v}^{+\infty} \int_{u}^{+\infty} \frac{1}{\alpha(u) \beta(v) \gamma(w)}
$$$$
\times\left[f\left(s, x\left(f_{1}(s)\right), \ldots, x\left(f_{k}(s)\right)\right)-g(s)\right] d s d u d v d w
$$$$
\leq L+\sum_{i=1}^{\infty} \int_{t+i \tau}^{+\infty} \int_{w}^{+\infty} \int_{v}^{+\infty} \frac{R_{2}(u)}{|\alpha(u) \beta(v) \gamma(w)|} d u d v d w
$$$$
+\sum_{i=1}^{\infty} \int_{t+i \tau}^{+\infty} \int_{w}^{+\infty} \int_{v}^{+\infty} \int_{u}^{+\infty} \frac{R_{1}(s)+|g(s)|}{|\alpha(u) \beta(v) \gamma(w)|} d s d u d v d w
$$$$
<L+\min \{M-L, L-N\}
$$$$
\leq M,
$$

$S_{L} x(t)$

$\geq L-\sum_{i=1}^{\infty} \int_{t+i \tau}^{+\infty} \int_{w}^{+\infty} \int_{v}^{+\infty} \frac{R_{2}(u)}{|\alpha(u) \beta(v) \gamma(w)|} d u d v d w$

$$
-\sum_{i=1}^{\infty} \int_{t+i \tau}^{+\infty} \int_{w}^{+\infty} \int_{v}^{+\infty} \int_{u}^{+\infty} \frac{R_{1}(s)+|g(s)|}{|\alpha(u) \beta(v) \gamma(w)|} d s d u d v d w
$$$$
>L-\min \{M-L, L-N\}
$$$$
\geq N \text {, }
$$

which yield that (29) holds. That is, $S_{L}$ is a contraction mapping in $A(N, M)$ and has a unique fixed point $x \in$ $A(N, M)$; that is,

$$
\begin{aligned}
& x(t) \\
& =L-\sum_{i=1}^{\infty} \int_{t+i \tau}^{+\infty} \int_{w}^{+\infty} \int_{v}^{+\infty} \frac{1}{\alpha(u) \beta(v) \gamma(w)} \\
& \times h\left(u, x\left(h_{1}(u)\right), \ldots, x\left(h_{k}(u)\right)\right) d u d v d w \\
& +\sum_{i=1}^{\infty} \int_{t+i \tau}^{+\infty} \int_{w}^{+\infty} \int_{v}^{+\infty} \int_{u}^{+\infty} \frac{1}{\alpha(u) \beta(v) \gamma(w)} \\
& \times\left[f\left(s, x\left(f_{1}(s)\right), \ldots, x\left(f_{k}(s)\right)\right)\right. \\
& -g(s)] d s d u d v d w, \quad t \geq T, \\
& x(t-\tau) \\
& =L-\sum_{i=1}^{\infty} \int_{t+(i-1) \tau}^{+\infty} \int_{w}^{+\infty} \int_{v}^{+\infty} \frac{1}{\alpha(u) \beta(v) \gamma(w)} \\
& \times h\left(u, x\left(h_{1}(u)\right), \ldots, x\left(h_{k}(u)\right)\right) d u d v d w \\
& +\sum_{i=1}^{\infty} \int_{t+(i-1) \tau}^{+\infty} \int_{w}^{+\infty} \int_{v}^{+\infty} \int_{u}^{+\infty} \frac{1}{\alpha(u) \beta(v) \gamma(w)} \\
& \times\left[f\left(s, x\left(f_{1}(s)\right), \ldots, x\left(f_{k}(s)\right)\right)\right. \\
& -g(s)] d s d u d v d w, \quad t \geq T+\tau .
\end{aligned}
$$

By virtue of (62) and (63), we get that

$$
\begin{aligned}
& x(t)-x(t-\tau) \\
& =\int_{t}^{+\infty} \int_{w}^{+\infty} \int_{v}^{+\infty} \frac{1}{\alpha(u) \beta(v) \gamma(w)} \\
& \times h\left(u, x\left(h_{1}(u)\right), \ldots, x\left(h_{k}(u)\right)\right) d u d v d w \\
& -\int_{t}^{+\infty} \int_{w}^{+\infty} \int_{v}^{+\infty} \int_{u}^{+\infty} \frac{1}{\alpha(u) \beta(v) \gamma(w)} \\
& \times\left[f\left(s, x\left(f_{1}(s)\right), \ldots, x\left(f_{k}(s)\right)\right)\right. \\
& \quad-g(s)] d s d u d v d w, \quad t \geq T+\tau,
\end{aligned}
$$

which yields that

$$
\begin{aligned}
(x(t) & -x(t-\tau))^{\prime} \\
= & -\int_{t}^{+\infty} \int_{v}^{+\infty} \frac{1}{\alpha(u) \beta(v) \gamma(t)} \\
& \times h\left(u, x\left(h_{1}(u)\right), \ldots, x\left(h_{k}(u)\right)\right) d u d v \\
& +\int_{t}^{+\infty} \int_{v}^{+\infty} \int_{u}^{+\infty} \frac{1}{\alpha(u) \beta(v) \gamma(t)} \\
& \times\left[f\left(s, x\left(f_{1}(s)\right), \ldots, x\left(f_{k}(s)\right)\right)\right. \\
& \quad-g(s)] d s d u d v, \quad t \geq T+\tau,
\end{aligned}
$$




$$
\begin{aligned}
& \left(\gamma(t)(x(t)-x(t-\tau))^{\prime}\right)^{\prime} \\
& =\int_{t}^{+\infty} \frac{1}{\alpha(u) \beta(t)} \\
& \quad \times h\left(u, x\left(h_{1}(u)\right), \ldots, x\left(h_{k}(u)\right)\right) d u \\
& \quad-\int_{t}^{+\infty} \int_{u}^{+\infty} \frac{1}{\alpha(u) \beta(t)} \\
& \quad \times\left[f\left(s, x\left(f_{1}(s)\right), \ldots, x\left(f_{k}(s)\right)\right)\right. \\
& \quad-g(s)] d s d u, \quad t \geq T+\tau,
\end{aligned}
$$

which ensures that

$$
\begin{aligned}
& {\left[\beta(t)\left(\gamma(t)(x(t)-x(t-\tau))^{\prime}\right)^{\prime}\right]^{\prime}} \\
& =-\frac{1}{\alpha(t)} h\left(u, x\left(h_{1}(u)\right), \ldots, x\left(h_{k}(u)\right)\right) \\
& \quad+\int_{t}^{+\infty} \frac{1}{\alpha(t)}\left[f\left(s, x\left(f_{1}(s)\right), \ldots, x\left(f_{k}(s)\right)\right)-g(s)\right] d s, \quad t \geq T+\tau, \\
& {\left[\alpha(t)\left[\beta(t)\left(\gamma(t)(x(t)-x(t-\tau))^{\prime}\right)^{\prime}\right]^{\prime}\right]^{\prime}} \\
& \quad=-\left[h\left(t, x\left(h_{1}(t)\right), \ldots, x\left(h_{k}(t)\right)\right)\right]^{\prime} \\
& \quad-\left[f\left(t, x\left(f_{1}(t)\right), \ldots, x\left(f_{k}(t)\right)\right)-g(t)\right], \\
& \quad t \geq T+\tau,
\end{aligned}
$$

which gives that $x$ is a bounded positive solution of (11). By means of (29), (57), (58), and (60), we deduce that, for any $m \in \mathbb{N}_{0}$ and $t \geq T$,

$$
\begin{aligned}
& \left|x_{m+1}(t)-x(t)\right| \\
& =\mid\left(1-\alpha_{m}-\beta_{m}\right) x_{m}(t)+\alpha_{m} \\
& \times\left\{L-\sum_{i=1}^{\infty} \int_{t+i \tau}^{+\infty} \int_{w}^{+\infty} \int_{v}^{+\infty} \frac{1}{\alpha(u) \beta(v) \gamma(w)}\right. \\
& \times h\left(u, x_{m}\left(h_{1}(u)\right), \ldots, x_{m}\left(h_{k}(u)\right)\right) d u d v d w \\
& +\sum_{i=1}^{\infty} \int_{t+i \tau}^{+\infty} \int_{w}^{+\infty} \int_{v}^{+\infty} \int_{u}^{+\infty} \frac{1}{\alpha(u) \beta(v) \gamma(w)} \\
& \times\left[f\left(s, x_{m}\left(f_{1}(s)\right), \ldots, x_{m}\left(f_{k}(s)\right)\right)\right. \\
& -g(s)] d s d u d v d w\}+\beta_{m} \gamma_{m}(t)-x(t) \mid \\
& \leq\left(1-\alpha_{m}-\beta_{m}\right)\left|x_{m}(t)-x(t)\right| \\
& +\alpha_{m}\left|S_{L} x_{m}(t)-S_{L} x(t)\right|+\beta_{m}\left|\gamma_{m}(t)-x(t)\right|
\end{aligned}
$$

$$
\begin{aligned}
\leq & \left(1-\alpha_{m}-\beta_{m}\right)\left|x_{m}(t)-x(t)\right| \\
& +\alpha_{m} \theta\left|x_{m}(t)-x(t)\right|+2 M \beta_{m} \\
\leq & \left(1-(1-\theta) \alpha_{m}\right)\left|x_{m}(t)-x(t)\right|+2 M \beta_{m},
\end{aligned}
$$

which implies (22). Thus Lemma 1, (23), and (24) ensure that $\lim _{m \rightarrow \infty} x_{m}=x$.

Next we show that (b) holds. Let $L_{1}, L_{2} \in(N, M)$ with $L_{1} \neq L_{2}$. As in the proof of (a), for each $j \in\{1,2\}$, we infer that there exists $\theta_{j} \in(0,1), T_{j}>t_{0}+|a|+\tau+\left|T_{0}\right|$, and $S_{L_{j}}$ : $A(N, M) \rightarrow A(N, M)$ satisfying (58)-(60), where $\theta, T, S_{L}$ are replaced by $\theta_{j}, T_{j}, S_{L_{j}}$, respectively, and the contraction mapping $S_{L_{j}}$ possesses a unique fixed point $z_{j} \in A(N, M)$, and $z_{j}$ is a bounded positive solution of (11); that is,

$$
\begin{aligned}
& z_{j}(t) \\
& =L_{j}-\sum_{i=1}^{\infty} \int_{t+i \tau}^{+\infty} \int_{w}^{+\infty} \int_{v}^{+\infty} \frac{1}{\alpha(u) \beta(v) \gamma(w)} \\
& \quad \times h\left(u, z_{j}\left(h_{1}(u)\right), \ldots, z_{j}\left(h_{k}(u)\right)\right) d u d v d w \\
& +\sum_{i=1}^{\infty} \int_{t+i \tau}^{+\infty} \int_{w}^{+\infty} \int_{v}^{+\infty} \int_{u}^{+\infty} \frac{1}{\alpha(u) \beta(v) \gamma(w)} \\
& \times\left[f\left(s, z_{j}\left(f_{1}(s)\right), \ldots, z_{j}\left(f_{k}(s)\right)\right)\right. \\
& \quad-g(s)] d s d u d v d w, \quad t \geq T_{j} .
\end{aligned}
$$

Put $T^{*}=\max \left\{T_{1}, T_{2}\right\}$. Using (16), (58), and (68), we conclude that for $t>T^{*}$

$$
\begin{aligned}
& \left|z_{1}(t)-z_{2}(t)\right| \\
& =\mid L_{1}-L_{2} \\
& -\sum_{i=1}^{\infty} \int_{t+i \tau}^{+\infty} \int_{w}^{+\infty} \int_{v}^{+\infty} \frac{1}{\alpha(u) \beta(v) \gamma(w)} \\
& \times\left[h\left(u, z_{1}\left(h_{1}(u)\right), \ldots, z_{1}\left(h_{k}(u)\right)\right)\right. \\
& \left.-h\left(u, z_{2}\left(h_{1}(u)\right), \ldots, z_{2}\left(h_{k}(u)\right)\right)\right] d u d v d w \\
& +\sum_{i=1}^{\infty} \int_{t+i \tau}^{+\infty} \int_{w}^{+\infty} \int_{v}^{+\infty} \int_{u}^{+\infty} \frac{1}{\alpha(u) \beta(v) \gamma(w)} \\
& \times\left[f\left(s, z_{1}\left(f_{1}(s)\right), \ldots, z_{1}\left(f_{k}(s)\right)\right)\right. \\
& \left.-f\left(s, z_{2}\left(f_{1}(s)\right), \ldots, z_{2}\left(f_{k}(s)\right)\right)\right] d s d u d v d w \mid \\
& \geq\left|L_{1}-L_{2}\right|-\left\|z_{1}-z_{2}\right\| \\
& \times \sum_{i=1}^{\infty} \int_{t+i \tau}^{+\infty} \int_{w}^{+\infty} \int_{v}^{+\infty} \frac{Q_{2}(u)}{|\alpha(u) \beta(v) \gamma(w)|} d u d v d w \\
& -\left\|z_{1}-z_{2}\right\| \\
& \times \sum_{i=1}^{\infty} \int_{t+i \tau}^{+\infty} \int_{w}^{+\infty} \int_{v}^{+\infty} \int_{u}^{+\infty} \frac{Q_{1}(s)}{|\alpha(u) \beta(v) \gamma(w)|} d s d u d v d w \\
& \geq\left|L_{1}-L_{2}\right|-\left\|z_{1}-z_{2}\right\| \\
& \times\left(\sum_{i=1}^{\infty} \int_{\max \left\{T_{1}, T_{2}\right\}+i \tau}^{+\infty} \int_{w}^{+\infty} \int_{v}^{+\infty} \frac{Q_{2}(u)}{|\alpha(u) \beta(v) \gamma(w)|} d u d v d w\right. \\
& \left.+\sum_{i=1}^{\infty} \int_{\max \left\{T_{1}, T_{2}\right\}+i \tau}^{+\infty} \int_{w}^{+\infty} \int_{v}^{+\infty} \int_{u}^{+\infty} \frac{Q_{1}(s)}{|\alpha(u) \beta(v) \gamma(w)|} d s d u d v d w\right) \\
& \geq\left|L_{1}-L_{2}\right|-\left\|z_{1}-z_{2}\right\| \max \left\{\theta_{1}, \theta_{2}\right\} \text {, }
\end{aligned}
$$


which yields that

$$
\left\|z_{1}-z_{2}\right\| \geq \frac{\left|L_{1}-L_{2}\right|}{1+\max \left\{\theta_{1}, \theta_{2}\right\}}>0 ;
$$

that is, $z_{1} \neq z_{2}$. This completes the proof.

Theorem 7. Assume that there exist constants $M, N, c_{1}, c_{2}$ and $T_{0} \geq t_{0}$ and functions $Q_{1}, Q_{2}, R_{1}$ and $R_{2} \in C\left(\left[t_{0},+\infty\right), \mathbb{R}^{+}\right)$ satisfying (16)-(18) and

$$
\begin{gathered}
1<c_{2}<c_{1}<c_{2}^{2}, \quad 0<\frac{c_{1}^{2}-c_{2}}{c_{1}} N<\frac{c_{2}^{2}-c_{1}}{c_{2}} M ; \\
c_{2} \leq p(t) \leq c_{1}, \quad t \geq T_{0} .
\end{gathered}
$$

Then

(a) for any $L \in\left(\left(c_{1} / c_{2}\right) M+c_{1} N,\left(c_{2} / c_{1}\right) N+c_{2} M\right)$, there exist $\theta \in(0,1)$ and $T>t_{0}+|a|+\tau+\left|T_{0}\right|$ such that, for each $x_{0} \in A(N, M)$, the Mann iterative sequence $\left\{x_{m}\right\}_{m \in \mathbb{N}_{0}}$ with mixed errors generated by the scheme

$$
x_{m+1}(t)=\left\{\begin{aligned}
(1- & \left.\alpha_{m}-\beta_{m}\right) x_{m}(t)+\alpha_{m} \\
\times & \left\{\frac{L}{p(t+\tau)}-\frac{x_{m}(t+\tau)}{p(t+\tau)}+\frac{1}{p(t+\tau)}\right. \\
& \times \int_{t+\tau}^{+\infty} \int_{w}^{+\infty} \int_{v}^{+\infty} \frac{1}{\alpha(u) \beta(v) \gamma(w)} \\
& \times h\left(u, x_{m}\left(h_{1}(u)\right), \ldots, x_{m}\left(h_{k}(u)\right)\right) d u d v d w \\
& -\frac{1}{p(t+\tau)} \\
& \times \int_{t+\tau}^{+\infty} \int_{w}^{+\infty} \int_{v}^{+\infty} \int_{u}^{+\infty} \frac{1}{\alpha(u) \beta(v) \gamma(w)} \\
& \times\left[f\left(s, x_{m}\left(f_{1}(s)\right), \ldots, x_{m}\left(f_{k}(s)\right)\right)\right. \\
& -g(s)] d s d u d v d w\}+\beta_{m} \gamma_{m}(t), \\
(1- & \left.\alpha_{m}-\beta_{m}\right) x_{m}(T)+\alpha_{m} \\
\times & \left\{\frac{L}{p(T+\tau)}-\frac{x_{m}(T+\tau)}{p(T+\tau)}+\frac{1}{p(T+\tau)}\right. \\
& \times \int_{T+\tau}^{+\infty} \int_{w}^{+\infty} \int_{v}^{+\infty} \overline{\alpha(u) \beta(v) \gamma(w)} \\
& \times h\left(u, x_{m}\left(h_{1}(u)\right), \ldots, x_{m}\left(h_{k}(u)\right)\right) d u d v d w \\
& -\frac{1}{p(T+\tau)} \int_{T+\tau}^{+\infty} \int_{w}^{+\infty} \int_{v}^{+\infty} \int_{u}^{+\infty} \overline{\alpha(u) \beta(v) \gamma(w)} \\
& \times\left[f\left(s, x_{m}\left(f_{1}(s)\right), \ldots, x_{m}\left(f_{k}(s)\right)\right)\right.
\end{aligned}\right.
$$

with (23) and (24) converges to a bounded positive solution $x \in A(N, M)$ of (11) and has the error estimate (22);

(b) equation (11) possesses uncountably many bounded positive solutions in $A(N, M)$.
Proof. In the first place, we prove that (a) holds. Let $L \in$ $\left(\left(c_{1} / c_{2}\right) M+c_{1} N,\left(c_{2} / c_{1}\right) N+c_{2} M\right)$. It follows from (18) and (71) that there exist $\theta \in(0,1)$ and $T>t_{0}+|a|+\tau+\left|T_{0}\right|$ satisfying

$\theta$

$$
\begin{aligned}
& =\frac{1}{c_{2}}\left(1+\int_{T}^{+\infty} \int_{w}^{+\infty} \int_{v}^{+\infty} \frac{Q_{2}(u)}{|\alpha(u) \beta(v) \gamma(w)|} d u d v d w\right. \\
& \left.\quad+\int_{T}^{+\infty} \int_{w}^{+\infty} \int_{v}^{+\infty} \int_{u}^{+\infty} \frac{Q_{1}(s)}{|\alpha(u) \beta(v) \gamma(w)|} d s d u d v d w\right), \\
& \int_{T}^{+\infty} \int_{w}^{+\infty} \int_{v}^{+\infty} \frac{R_{2}(u)}{|\alpha(u) \beta(v) \gamma(w)|} d u d v d w \\
& \quad+\int_{T}^{+\infty} \int_{w}^{+\infty} \int_{v}^{+\infty} \int_{u}^{+\infty} \frac{R_{1}(s)+|g(s)|}{|\alpha(u) \beta(v) \gamma(w)|} d s d u d v d w \\
& <\min \left\{c_{2} M-L+\frac{c_{2}}{c_{1}} N,-c_{2} N+\frac{c_{2}}{c_{1}} L-M\right\} .
\end{aligned}
$$

Define a mapping $S_{L}: A(N, M) \rightarrow C B([a,+\infty), \mathbb{R})$ by

$$
S_{L} x(t)=\left\{\begin{array}{c}
\frac{L}{p(t+\tau)}-\frac{x(t+\tau)}{p(t+\tau)}+\frac{1}{p(t+\tau)} \\
\quad \times \int_{t+\tau}^{+\infty} \int_{w}^{+\infty} \int_{v}^{+\infty} \frac{1}{\alpha(u) \beta(v) \gamma(w)} \\
\quad \times h\left(u, x\left(h_{1}(u)\right), \ldots, x\left(h_{k}(u)\right)\right) d u d v d w \\
\quad \frac{1}{p(t+\tau)} \\
\quad \times \int_{t+\tau}^{+\infty} \int_{w}^{+\infty} \int_{v}^{+\infty} \int_{u}^{+\infty} \frac{1}{\alpha(u) \beta(v) \gamma(w)} \\
\times\left[f\left(s, x\left(f_{1}(s)\right), \ldots, x\left(f_{k}(s)\right)\right)\right. \\
-g(s)] d s d u d v d w, \\
t \geq T, \quad x \in A(N, M), \\
S_{L} x(T), \quad a \leq t<T, x \in A(N, M) .
\end{array}\right.
$$

Obviously, $S_{L} x$ is continuous for each $x \in A(N, M)$. In view of (16), (17), (71), (72), and (74)-(76), we conclude that for $x, y \in A(N, M)$ and $t \geq T$

$$
\begin{aligned}
& \left|S_{L} x(t)-S_{L} y(t)\right| \\
& \leq \frac{1}{|p(t+\tau)|}|x(t+\tau)-y(t+\tau)|+\frac{1}{|p(t+\tau)|} \\
& \quad \times \int_{t+\tau}^{+\infty} \int_{w}^{+\infty} \int_{v}^{+\infty} \frac{1}{|\alpha(u) \beta(v) \gamma(w)|} \\
& \quad \times \mid h\left(u, x\left(h_{1}(u)\right), \ldots, x\left(h_{k}(u)\right)\right) \\
& \quad-h\left(u, y\left(h_{1}(u)\right), \ldots, y\left(h_{k}(u)\right)\right) \mid d u d v d w \\
& +\frac{1}{|p(t+\tau)|} \int_{t+t}^{+\infty} \int_{w}^{+\infty} \int_{v}^{+\infty} \int_{u}^{+\infty} \frac{1}{|\alpha(u) \beta(v) \gamma(w)|} \\
& \quad \times \mid f\left(s, x\left(f_{1}(s)\right), \ldots, x\left(f_{k}(s)\right)\right) \\
& \quad-f\left(s, y\left(f_{1}(s)\right), \ldots, y\left(f_{k}(s)\right)\right) \mid d s d u d v d w
\end{aligned}
$$




$$
\begin{array}{lll}
\leq \frac{1}{c_{2}}\|x-y\| & >\frac{L}{c_{1}}-\frac{M}{c_{2}}-\frac{1}{c_{2}} \\
\times\left(1+\int_{T}^{+\infty} \int_{w}^{+\infty} \int_{v}^{+\infty} \frac{Q_{2}(u)}{|\alpha(u) \beta(v) \gamma(w)|} d u d v d w\right. & \times \min \left\{c_{2} M-L+\frac{c_{2}}{c_{1}} N,-c_{2} N+\frac{c_{2}}{c_{1}} L-M\right\} \\
& \left.+\int_{T}^{+\infty} \int_{w}^{+\infty} \int_{v}^{+\infty} \int_{u}^{+\infty} \frac{Q_{1}(s)}{|\alpha(u) \beta(v) \gamma(w)|} d s d u d v d w\right) & \geq N,
\end{array}
$$

$=\theta\|x-y\|$,

$S_{L} x(t)$

$$
\begin{aligned}
& =\frac{L}{p(t+\tau)}-\frac{x(t+\tau)}{p(t+\tau)} \\
& +\frac{1}{p(t+\tau)} \int_{t+\tau}^{+\infty} \int_{w}^{+\infty} \int_{v}^{+\infty} \frac{1}{\alpha(u) \beta(v) \gamma(w)} \\
& \times h\left(u, x\left(h_{1}(u)\right), \ldots, x\left(h_{k}(u)\right)\right) d u d v d w \\
& -\frac{1}{p(t+\tau)} \int_{t+\tau}^{+\infty} \int_{w}^{+\infty} \int_{v}^{+\infty} \int_{u}^{+\infty} \frac{1}{\alpha(u) \beta(v) \gamma(w)} \\
& \times\left[f\left(s, x\left(f_{1}(s)\right), \ldots, x\left(f_{k}(s)\right)\right)\right. \\
& \leq \frac{L}{c_{2}}-\frac{N}{c_{1}}+\frac{1}{c_{2}} \\
& \left.\times \frac{L}{c_{2}}-\frac{N}{c_{1}}+\frac{1}{c_{2}}(s)\right] d s d u d v d w \\
& \times \int_{T}^{+\infty} \int_{w}^{+\infty} \int_{v}^{+\infty} \frac{R_{2}(u)}{|\alpha(u) \beta(v) \gamma(w)|} d u d v d w \\
&
\end{aligned}
$$$$
\leq M
$$$$
S_{L} x(t)
$$$$
\geq \frac{L}{c_{1}}-\frac{M}{c_{2}}-\frac{1}{c_{2}}
$$$$
\times \int_{T}^{+\infty} \int_{w}^{+\infty} \int_{v}^{+\infty} \frac{R_{2}(u)}{|\alpha(u) \beta(v) \gamma(w)|} d u d v d w
$$$$
-\frac{1}{c_{2}} \int_{T}^{+\infty} \int_{w}^{+\infty} \int_{v}^{+\infty} \int_{u}^{+\infty} \frac{R_{1}(s)+|g(s)|}{|\alpha(u) \beta(v) \gamma(w)|} d s d u d v d w
$$

which imply that (29) holds. That is, $S_{L}$ is a contraction mapping in $A(N, M)$ and has a unique fixed point $x \in$ $A(N, M)$, which is a bounded positive solution of (11). By means of (29), (73), and (76), we obtain that for any $m \in \mathbb{N}_{0}$ and $t \geq T$

$$
\begin{aligned}
& \left|x_{m+1}(t)-x(t)\right| \\
& =\mid\left(1-\alpha_{m}-\beta_{m}\right) x_{m}(t)+\alpha_{m} \\
& \times\left\{\frac{L}{p(t+\tau)}-\frac{x(t+\tau)}{p(t+\tau)}+\frac{1}{p(t+\tau)}\right. \\
& \times \int_{t+\tau}^{+\infty} \int_{w}^{+\infty} \int_{v}^{+\infty} \frac{1}{\alpha(u) \beta(v) \gamma(w)} \\
& \times h\left(u, x_{m}\left(h_{1}(u)\right), \ldots, x_{m}\left(h_{k}(u)\right)\right) d u d v d w \\
& -\frac{1}{p(t+\tau)} \int_{t+\tau}^{+\infty} \int_{w}^{+\infty} \int_{v}^{+\infty} \int_{u}^{+\infty} \frac{1}{\alpha(u) \beta(v) \gamma(w)} \\
& \times\left[f\left(s, x_{m}\left(f_{1}(s)\right), \ldots, x_{m}\left(f_{k}(s)\right)\right)\right. \\
& -g(s)] d s d u d v d w\}+\beta_{m} \gamma_{m}(t)-x(t) \\
& \leq\left(1-\alpha_{m}-\beta_{m}\right)\left|x_{m}(t)-x(t)\right| \\
& +\alpha_{m}\left|S_{L} x_{m}(t)-S_{L} x(t)\right|+\beta_{m}\left|\gamma_{m}(t)-x(t)\right| \\
& \leq\left(1-\alpha_{m}-\beta_{m}\right)\left|x_{m}(t)-x(t)\right| \\
& +\alpha_{m} \theta\left|x_{m}(t)-x(t)\right|+2 M \beta_{m} \\
& \leq\left(1-(1-\theta) \alpha_{m}\right)\left|x_{m}(t)-x(t)\right|+2 M \beta_{m} \\
& \leq\left(1-(1-\theta) \alpha_{m}\right)\left\|x_{m}-x\right\|+2 M \beta_{m},
\end{aligned}
$$

which gives (22). Thus Lemma 1, (23), and (24) mean that $\lim _{m \rightarrow \infty} x_{m}=x$.

Next we show that (b) holds. Let $L_{1}, L_{2} \in\left(\left(c_{1} / c_{2}\right) M+\right.$ $\left.c_{1} N,\left(c_{2} / c_{1}\right) N+c_{2} M\right)$ with $L_{1} \neq L_{2}$. As in the proof of (a), we conclude that, for each $\in\{1,2\}$, there exist $\theta_{j} \in(0,1), T_{j}>$ $t_{0}+|a|+\tau+\left|T_{0}\right|$ and $S_{L_{j}}: A(N, M) \rightarrow A(N, M)$ satisfying (74)-(76), where $\theta, T, S_{L}$ are replaced by $\theta_{j}, T_{j}$, 
and $S_{L_{j}}$, respectively, and the contraction mapping $S_{L_{j}}$ has a unique fixed point $z_{j} \in A(N, M)$, which is also a bounded positive solution of (11). In order to prove (b), we need only to show that $z_{1} \neq z_{2}$. Put $T^{*}=\max \left\{T_{1}, T_{2}\right\}$. Note that for $t \geq T^{*}$ and $j \in\{1,2\}$

$$
\begin{aligned}
& z_{j}(t) \\
& =\frac{L_{j}}{p(t+\tau)}-\frac{z_{j}(t+\tau)}{p(t+\tau)}+\frac{1}{p(t+\tau)} \\
& \quad \times \int_{t+\tau}^{+\infty} \int_{w}^{+\infty} \int_{v}^{+\infty} \frac{1}{\alpha(u) \beta(v) \gamma(w)} \\
& \times h\left(u, z_{j}\left(h_{1}(u)\right), \ldots, z_{j}\left(h_{k}(u)\right)\right) d u d v d w \\
& -\frac{1}{p(t+\tau)} \\
& \quad \times \int_{t+\tau}^{+\infty} \int_{w}^{+\infty} \int_{v}^{+\infty} \int_{u}^{+\infty} \frac{1}{\alpha(u) \beta(v) \gamma(w)} \\
& \quad \times\left[f\left(s, z_{j}\left(f_{1}(s)\right), \ldots, z_{j}\left(f_{k}(s)\right)\right)\right. \\
& \quad-g(s)] d s d u d v d w,
\end{aligned}
$$

which together with (16) and (74) implies that for $t \geq T^{*}$

$$
\begin{aligned}
& \left|z_{1}(t)-z_{2}(t)\right| \\
& =\mid \frac{L_{1}-L_{2}}{p(t+\tau)}-\frac{z_{1}(t+\tau)-z_{2}(t+\tau)}{p(t+\tau)}+\frac{1}{p(t+\tau)} \\
& \quad \times \int_{t+\tau}^{+\infty} \int_{w}^{+\infty} \int_{v}^{+\infty} \frac{1}{\alpha(u) \beta(v) \gamma(w)} \\
& \quad \times\left[h\left(u, z_{1}\left(h_{1}(u)\right), \ldots, z_{1}\left(h_{k}(u)\right)\right)\right. \\
& \quad-\frac{1}{p(t+\tau)} \\
& \quad \times \int_{t+\tau}^{+\infty} \int_{w}^{+\infty} \int_{v}^{+\infty} \int_{u}^{+\infty} \frac{1}{\alpha(u) \beta(v) \gamma(w)} \\
& \quad \times\left[f\left(s, z_{1}\left(f_{1}(s)\right), \ldots, z_{1}\left(f_{k}(s)\right)\right)\right. \\
& \left.\left.\left.\quad-\frac{\left\|z_{1}-z_{2}\right\|}{c_{2}}(u)\right), \ldots, z_{2}\left(h_{k}(u)\right)\right)\right] d u d v d w \\
& \quad \times \frac{\left|L_{1}-L_{2}\right|}{c_{1}}-\frac{\left\|z_{1}-z_{2}\right\|}{c_{2}}-\frac{\left\|z_{1}-z_{2}\right\|}{c_{2}} \int_{v}^{+\infty} \frac{Q_{2}(u)}{|\alpha(u) \beta(v) \gamma(w)|} d u d v d w \\
& \left.\quad-f\left(s, z_{2}\left(f_{1}(s)\right), \ldots, z_{2}\left(f_{k}(s)\right)\right)\right] d s d u d v d w \mid
\end{aligned}
$$

$$
\begin{aligned}
& \times \int_{T^{*}}^{+\infty} \int_{w}^{+\infty} \int_{v}^{+\infty} \int_{u}^{+\infty} \frac{Q_{1}(s)}{|\alpha(u) \beta(v) \gamma(w)|} d s d u d v d w \\
\geq & \frac{\left|L_{1}-L_{2}\right|}{c_{1}}-\left\|z_{1}-z_{2}\right\| \max \left\{\theta_{1}, \theta_{2}\right\},
\end{aligned}
$$

which yields that

$$
\left\|z_{1}-z_{2}\right\| \geq \frac{\left|L_{1}-L_{2}\right|}{c_{1}\left(1+\max \left\{\theta_{1}, \theta_{2}\right\}\right)}>0
$$

that is, $z_{1} \neq z_{2}$. This completes the proof.

Theorem 8. Assume that there exist constants $M, N, c_{1}, c_{2}$ and $T_{0} \geq t_{0}$ and functions $Q_{1}, Q_{2}, R_{1}$ and $R_{2} \in C\left(\left[t_{0},+\infty\right), \mathbb{R}^{+}\right)$ satisfying (16)-(18) and

$$
\begin{gathered}
0<\left(c_{1}-1\right) N<\left(c_{2}-1\right) M \\
-\infty<-c_{1} \leq p(t) \leq-c_{2}<-1, \quad t \geq T_{0} .
\end{gathered}
$$

Then

(a) for any $L \in\left(\left(c_{1}-1\right) N,\left(c_{2}-1\right) M\right)$, there exist $\theta \in$ $(0,1)$ and $T>t_{0}+|a|+\tau+\left|T_{0}\right|$ such that, for each $x_{0} \in A(N, M)$, the Mann iterative sequence $\left\{x_{m}\right\}_{m \in \mathbb{N}_{0}}$ with mixed errors generated by the scheme

$$
x_{m+1}(t)=\left\{\begin{aligned}
&\left(1-\alpha_{m}-\beta_{m}\right) x_{m}(t)+\alpha_{m} \\
& \times\left\{\frac{-L}{p(t+\tau)}-\frac{x_{m}(t+\tau)}{p(t+\tau)}+\frac{1}{p(t+\tau)}\right. \\
& \times \int_{t+\tau}^{+\infty} \int_{w}^{+\infty} \int_{v}^{+\infty} \frac{1}{\alpha(u) \beta(v) \gamma(w)} \\
& \times h\left(u, x_{m}\left(h_{1}(u)\right), \ldots, x_{m}\left(h_{k}(u)\right)\right) d u d v d w \\
&-\frac{1}{p(t+\tau)} \int_{t+\tau}^{+\infty} \int_{w}^{+\infty} \int_{v}^{+\infty} \int_{u}^{+\infty} \frac{1}{\alpha(u) \beta(v) \gamma(w)} \\
& \times\left[f\left(s, x_{m}\left(f_{1}(s)\right), \ldots, x_{m}\left(f_{k}(s)\right)\right)\right. \\
&(1-\left.\alpha_{m}-\beta_{m}\right) x_{m}(T)+\alpha_{m} \\
& \times\left\{\frac{-L}{p(T+\tau)}-\frac{x_{m}(T+\tau)}{p(T+\tau)}+\frac{1}{p(T+\tau)}\right. \\
& \times \int_{T+\tau}^{+\infty} \int_{w}^{+\infty} \int_{v}^{+\infty} \frac{1}{\alpha(u) \beta(v) \gamma(w)} \\
& \times h\left(u, x_{m}\left(h_{1}(u)\right), \ldots, x_{m}\left(h_{k}(u)\right)\right) d u d v d w \\
&-\frac{1}{p(T+\tau)} \quad \\
& \times \int_{T+\tau}^{+\infty} \int_{w}^{+\infty} \int_{v}^{+\infty} \int_{u}^{+\infty} \frac{1}{\alpha(u) \beta(v) \gamma(w)} \\
& \times\left[f\left(s, x_{m}\left(f_{1}(s)\right), \ldots, x_{m}\left(f_{k}(s)\right)\right)\right.
\end{aligned}\right.
$$

with (23) and (24) converges to a bounded positive solution $x \in A(N, M)$ of (11) and has the error estimate (22);

(b) equation (11) possesses uncountably many bounded positive solutions in $A(N, M)$. 
Proof. First of all, we prove that (a) holds. Let $L \in\left(\left(c_{1}-\right.\right.$ 1) $\left.N,\left(c_{2}-1\right) M\right)$. It follows from (18) and (82) that there exist $\theta \in(0,1)$ and $T>t_{0}+|a|+\tau+\left|T_{0}\right|$ satisfying (74) and

$$
\begin{aligned}
& \int_{T}^{+\infty} \int_{w}^{+\infty} \int_{v}^{+\infty} \frac{R_{2}(u)}{|\alpha(u) \beta(v) \gamma(w)|} d u d v d w \\
& \quad+\int_{T}^{+\infty} \int_{w}^{+\infty} \int_{v}^{+\infty} \int_{u}^{+\infty} \frac{R_{1}(s)+|g(s)|}{|\alpha(u) \beta(v) \gamma(w)|} d s d u d v d w \\
& <\min \left\{\left(c_{2}-1\right) M-L,\left(\frac{c_{2}}{c_{1}}-c_{2}\right) N+\frac{c_{2}}{c_{1}} L\right\} .
\end{aligned}
$$

Define a mapping $S_{L}: A(N, M) \rightarrow C B([a,+\infty), \mathbb{R})$ by

$$
S_{L} x(t)=\left\{\begin{array}{c}
\frac{-L}{p(t+\tau)}-\frac{x(t+\tau)}{p(t+\tau)}+\frac{1}{p(t+\tau)} \\
\quad \times \int_{t+\tau}^{+\infty} \int_{w}^{+\infty} \int_{v}^{+\infty} \frac{1}{\alpha(u) \beta(v) \gamma(w)} \\
\quad \times h\left(u, x\left(h_{1}(u)\right), \ldots, x\left(h_{k}(u)\right)\right) d u d v d w \\
-\frac{1}{p(t+\tau)} \\
\times \int_{t+\tau}^{+\infty} \int_{w}^{+\infty} \int_{v}^{+\infty} \int_{u}^{+\infty} \frac{1}{\alpha(u) \beta(v) \gamma(w)} \\
\quad \times\left[f\left(s, x\left(f_{1}(s)\right), \ldots, x\left(f_{k}(s)\right)\right)\right. \\
\quad-g(s)] d s d u d v d w, \\
t \geq T, x \in A(N, M), \\
S_{L} x(T), \quad a \leq t<T, x \in A(N, M) .
\end{array}\right.
$$

Obviously $S_{L} x$ is continuous for each $x \in A(N, M)$. On account of (17), (82), (84), and (85), we get that for any $x \in$ $A(N, M)$ and $t \geq T$

$$
\begin{aligned}
& S_{L} x(t) \\
& =\frac{-L}{p(t+\tau)}-\frac{x(t+\tau)}{p(t+\tau)}+\frac{1}{p(t+\tau)} \\
& \quad \times \int_{t+\tau}^{+\infty} \int_{w}^{+\infty} \int_{v}^{+\infty} \frac{1}{\alpha(u) \beta(v) \gamma(w)} \\
& \quad \times h\left(u, x\left(h_{1}(u)\right), \ldots, x\left(h_{k}(u)\right)\right) d u d v d w \\
& \quad-\frac{1}{p(t+\tau)} \\
& \quad \times \int_{t+\tau}^{+\infty} \int_{w}^{+\infty} \int_{v}^{+\infty} \int_{u}^{+\infty} \frac{1}{\alpha(u) \beta(v) \gamma(w)}
\end{aligned}
$$

$$
\begin{aligned}
& \times\left[f\left(s, x\left(f_{1}(s)\right), \ldots, x\left(f_{k}(s)\right)\right)-g(s)\right] d s d u d v d w \\
& \leq \frac{L}{c_{2}}+\frac{M}{c_{2}}+\frac{1}{c_{2}} \\
& \times \int_{T}^{+\infty} \int_{w}^{+\infty} \int_{v}^{+\infty} \frac{R_{2}(u)}{|\alpha(u) \beta(v) \gamma(w)|} d u d v d w \\
&+\frac{1}{c_{2}} \\
& \times \int_{T}^{+\infty} \int_{w}^{+\infty} \int_{v}^{+\infty} \int_{u}^{+\infty} \frac{R_{1}(s)+|g(s)|}{|\alpha(u) \beta(v) \gamma(w)|} d s d u d v d w \\
&< \frac{L}{c_{2}}+\frac{M}{c_{2}}+\frac{1}{c_{2}} \\
& \quad \times \min \left\{\left(c_{2}-1\right) M-L,\left(\frac{c_{2}}{c_{1}}-c_{2}\right) N+\frac{c_{2}}{c_{1}} L\right\}
\end{aligned}
$$$$
\leq M,
$$$$
S_{L} x(t)
$$$$
\geq \frac{L}{c_{1}}+\frac{N}{c_{1}}-\frac{1}{c_{2}}
$$$$
\times \int_{T}^{+\infty} \int_{w}^{+\infty} \int_{v}^{+\infty} \frac{R_{2}(u)}{|\alpha(u) \beta(v) \gamma(w)|} d u d v d w
$$$$
-\frac{1}{c_{2}}
$$$$
\times \int_{T}^{+\infty} \int_{w}^{+\infty} \int_{v}^{+\infty} \int_{u}^{+\infty} \frac{R_{1}(s)+|g(s)|}{|\alpha(u) \beta(v) \gamma(w)|} d s d u d v d w
$$$$
>\frac{L}{c_{1}}+\frac{N}{c_{1}}-\frac{1}{c_{2}}
$$$$
\times \min \left\{\left(c_{2}-1\right) M-L,\left(\frac{c_{2}}{c_{1}}-c_{2}\right) N+\frac{c_{2}}{c_{1}} L\right\}
$$$$
\geq N
$$

which imply (29). That is, $S_{L}$ is a contraction mapping in $A(N, M)$ and has a unique fixed point $x \in A(N, M)$, which is a bounded positive solution of (11). The rest of the proof is similar to that of Theorem 7 and is omitted. This completes the proof.

\section{Examples}

Now we construct seven examples as applications of the results presented in Section 2. 
Example 9. Consider the following fourth order nonlinear neutral delay differential equation:

$$
\begin{aligned}
& \left(\left(t^{2}+1\right)\right. \\
& \times\left(\frac{2 t^{4}+1}{\ln (t+1)}\right. \\
& \quad \times\left(-t^{3}\left(x(t)+\frac{3 t^{2} \cos t-1 / 2}{\left.\left.\left.\left.1+10 t^{2} x(t-\tau)\right)^{\prime}\right)^{\prime}\right)^{\prime}\right)^{\prime}}\right)^{\prime}\right. \\
& +\left(\frac{t \sin ^{2}\left(t x^{3}(t-1)\right)}{(t+3)^{2}(t+1)^{3}}\right)^{\prime}+\frac{\cos (t) x^{3}\left(t^{2}\right)}{t^{3}} \\
& =\frac{\sin \left(2-t^{2}\right)+1}{3 t^{4}}, \quad t \geq 3,
\end{aligned}
$$

where $\tau>0, t_{0}=T_{0}=3, k=1, N=2, M=20, c_{1}=3 / 10$, $c_{2}=3 / 5, a=\min \{3-\tau, 2\}$, and

$$
\begin{gathered}
\alpha(t)=t^{2}+1, \quad \beta(t)=\frac{2 t^{4}+1}{\ln (t+1)}, \\
\gamma(t)=-t^{3}, \quad p(t)=\frac{3 t^{2} \cos t-1 / 2}{1+10 t^{2}}, \\
g(t)=\frac{\sin \left(2-t^{2}\right)+1}{3 t^{4}}, \quad h_{1}(t)=t-1, \\
f_{1}(t)=t^{2}, \quad f(t, u)=\frac{u^{3} \cos (t)}{t^{3}}, \\
h(t, u)=\frac{t \sin ^{2}\left(t u^{3}\right)}{(t+3)^{2}(t+1)^{3}}, \quad Q_{1}(t)=\frac{3 M^{2}}{t^{3}}, \\
Q_{2}(t)=\frac{6 t^{2} M^{2}}{(t+3)^{2}(t+1)^{3}}, \quad R_{1}(t)=\frac{M^{3}}{t^{3}}, \\
R_{2}(t)=\frac{t}{(t+3)^{2}(t+1)^{3}}, \quad(t, u) \in\left[t_{0},+\infty\right) \times \mathbb{R} .
\end{gathered}
$$

It is easy to verify that (16)-(20) are satisfied. It follows from Theorem 2 that (11) possesses uncountably many bounded positive solutions in $A(N, M)$. On the other hand, for any $L \in\left(N+c_{1} M,\left(1-c_{2}\right) M\right)$, there exist $\theta \in(0,1)$ and $T>$ $t_{0}+|a|+\tau+\left|T_{0}\right|$ such that the Mann iterative sequence $\left\{x_{m}\right\}_{m \in \mathbb{N}_{0}}$ with mixed errors generated by (21) converges to a bounded positive solution $x \in A(N, M)$ of (11) and has the error estimate (22), where $\left\{\gamma_{m}\right\}_{m \in \mathbb{N}_{0}}$ is an arbitrary sequence in $A(N, M)$, and $\left\{\alpha_{m}\right\}_{m \in \mathbb{N}_{0}}$ and $\left\{\beta_{m}\right\}_{m \in \mathbb{N}_{0}}$ are any sequences in $[0,1]$ satisfying $(23)$ and $(24)$.
Example 10. Consider the following fourth order nonlinear neutral delay differential equation:

$$
\begin{aligned}
& \left(\frac{t^{6} \ln \left(1+t^{2}\right)}{1+t}\right. \\
& \left.\quad \times\left(t^{4}\left(-\sqrt{t^{7}+1}\left(x(t)+\frac{t^{3}}{3 t^{3}+2} x(t-\tau)\right)^{\prime}\right)^{\prime}\right)^{\prime}\right)^{\prime} \\
& +\left(\frac{x^{2}(t-2)+x^{2}(\sqrt{t+1})-x(\sqrt{t+1})}{1+t^{3}}\right)^{\prime} \\
& +\frac{2 x^{2}(t-1)+x^{2}(t-\sqrt{t})}{2 t^{4}+1}=\frac{1-t}{t^{4}+\cos ^{2} t}, \quad t \geq 10
\end{aligned}
$$

where $\tau>0, t_{0}=T_{0}=10, k=2, N=5, M=21, c_{1}=1 / 3$, $c_{2}=1 / 4, a=\min \{10-\tau, \sqrt{11}\}$, and

$$
\begin{gathered}
\alpha(t)=\frac{t^{6} \ln \left(1+t^{2}\right)}{1+t}, \quad \beta(t)=t^{4}, \\
\gamma(t)=-\sqrt{t^{7}+1}, \quad p(t)=\frac{t^{3}}{3 t^{3}+2}, \\
g(t)=\frac{1-t}{t^{4}+\cos ^{2} t}, \quad h_{1}(t)=t-2, \\
h_{2}(t)=\sqrt{t+1}, \quad f_{1}(t)=t-1, \\
f_{2}(t)=t-\sqrt{t}, \quad f(t, u, v)=\frac{2 u^{2}+v^{2}}{2 t^{4}+1}, \\
Q_{1}(t)=\frac{6 M}{2 t^{4}+1}, \quad Q Q_{2}(t)=\frac{4 M+1}{1+t^{3}}, \\
R_{2}(t)=\frac{u^{2}+v^{2}-v}{1+M^{2}+M}, \quad(t)=\frac{3 M^{2}}{2 t^{4}+1}, \\
(t, u, v) \in\left[t_{0},+\infty\right) \times \mathbb{R}^{2} .
\end{gathered}
$$

It is easy to verify that (16)-(18) and (35) are satisfied. It follows from Theorem 3 that (11) possesses uncountably many bounded positive solutions in $A(N, M)$. On the other hand, for any $L \in\left(N+c_{1} M, M+c_{2} N\right)$, there exist $\theta \in$ $(0,1)$ and $T>t_{0}+|a|+\tau+\left|T_{0}\right|$ such that the Mann iterative sequence $\left\{x_{m}\right\}_{m \in \mathbb{N}_{0}}$ with mixed errors generated by (21) converges to a bounded positive solution $x \in A(N, M)$ of (11) and has the error estimate (22), where $\left\{\gamma_{m}\right\}_{m \in \mathbb{N}_{0}}$ is an arbitrary sequence in $A(N, M)$, and $\left\{\alpha_{m}\right\}_{m \in \mathbb{N}_{0}}$ and $\left\{\beta_{m}\right\}_{m \in \mathbb{N}_{0}}$ are any sequences in $[0,1]$ satisfying (23) and (24). 
Example 11. Consider the following fourth order nonlinear neutral delay differential equation:

$$
\begin{aligned}
& \left(-t^{5}\left(\left(3 t^{2}+2\right)\right.\right. \\
& \left.\left.\quad \times\left(\frac{(2 t+1)^{3}}{t}\left(x(t)+\frac{1-t}{3+5 t} x(t-\tau)\right)^{\prime}\right)^{\prime}\right)^{\prime}\right)^{\prime} \\
& +\left(\frac{t x^{2}(2 t-3) x^{2}\left(e^{t}\right)}{\left(1+t^{2}\right)\left(2+\sin \left(x(2 t-3) x\left(t^{2}\right)\right)\right)}\right)^{\prime} \\
& +\frac{t^{2}}{1+t^{7}} x^{2}\left((t+1)^{2}\right) \\
& +\frac{\sqrt{\ln (t+1)}}{e^{t}+1} \sin ^{2}\left(t x(3 t+1) x^{3}(2 t)\right) \\
& =\frac{\sqrt{1+t^{2}}}{t^{4}+1}, \quad t \geq 2
\end{aligned}
$$

where $\tau>0, t_{0}=T_{0}=2, k=3, N=100, M=500, c_{1}=4 / 5$, $c_{2}=1 / 13, a=\min \{2-\tau, 1\}$, and

$$
\begin{aligned}
& \alpha(t)=-t^{5}, \quad \beta(t)=3 t^{2}+2, \\
& \gamma(t)=\frac{(2 t+1)^{3}}{t}, \quad p(t)=\frac{1-t}{3+5 t}, \\
& g(t)=\frac{\sqrt{1+t^{2}}}{t^{4}+1}, \quad h_{1}(t)=2 t-3, \\
& h_{2}(t)=t^{2}, \quad h_{3}(t)=e^{t}, \\
& f_{1}(t)=(t+1)^{2}, \quad f_{2}(t)=3 t+1, \quad f_{3}(t)=2 t, \\
& f(t, u, v, w)=\frac{t^{2} u^{2}}{1+t^{7}}+\frac{\sqrt{\ln (t+1)}}{e^{t}+1} \sin ^{2}\left(t v w^{3}\right), \\
& h(t, u, v, w)=\frac{t u^{2} w^{2}}{\left(1+t^{2}\right)(2+\sin (u v))}, \\
& Q_{1}(t)=\frac{2 M t^{2}}{1+t^{7}}+\frac{4 t M^{3} \sqrt{\ln (t+1)}}{e^{t}+1}, \\
& Q_{2}(t)=\frac{t\left(2 M^{5}+12 M^{3}\right)}{1+t^{2}}, \\
& R_{1}(t)=\frac{t^{2} M^{2}}{1+t^{7}}+\frac{\sqrt{\ln (t+1)}}{e^{t}+1} \\
& R_{2}(t)=\frac{t M^{4}}{1+t^{2}}, \quad(t, u, v, w) \in\left[t_{0},+\infty\right) \times \mathbb{R}^{3} .
\end{aligned}
$$

It is easy to verify that (17), (18), and (39) are satisfied. It follows from Theorem 4 that (11) possesses uncountably many bounded positive solutions in $A(N, M)$. On the other hand, for any $L \in\left(\left(1-c_{2}\right) N,\left(1-c_{1}\right) M\right)$, there exist $\theta \in(0,1)$ and $T>t_{0}+|a|+\tau+\left|T_{0}\right|$ such that the Mann iterative sequence $\left\{x_{m}\right\}_{m \in \mathbb{N}_{0}}$ with mixed errors generated by (21) converges to a bounded positive solution $x \in A(N, M)$ of (11) and has the error estimate (22), where $\left\{\gamma_{m}\right\}_{m \in \mathbb{N}_{0}}$ is an arbitrary sequence in $A(N, M)$, and $\left\{\alpha_{m}\right\}_{m \in \mathbb{N}_{0}}$ and $\left\{\beta_{m}\right\}_{m \in \mathbb{N}_{0}}$ are any sequences in $[0,1]$ satisfying $(23)$ and $(24)$.

Example 12. Consider the following fourth order nonlinear neutral delay differential equation:

$$
\begin{gathered}
\left(\frac{2 t^{4}}{1+t}\left(\left(t^{2}+1\right)\left(-t^{8}(x(t)+x(t-\tau))^{\prime}\right)^{\prime}\right)^{\prime}\right)^{\prime} \\
+\left(\frac{t^{3}+1}{t^{10} \ln (t+2)} x^{2}(t-2) x(t-1)\right)^{\prime} \\
+\frac{x^{2}(t-3)}{\left(1+t^{3}\right)\left(1+x^{2}(t-4)\right)} \\
=\frac{\sqrt{1+t^{2}}-\sin \left(2 t^{2}+1\right)}{t^{8}}, \quad t \geq 3,
\end{gathered}
$$

where $\tau>0, t_{0}=T_{0}=3, k=2, N=10, M=20, a=$ $\min \{3-\tau,-1\}$, and

$$
\begin{gathered}
\alpha(t)=\frac{2 t^{4}}{1+t}, \quad \beta(t)=t^{2}+1, \\
\gamma(t)=-t^{8}, \quad p(t)=1, \\
g(t)=\frac{\sqrt{1+t^{2}}-\sin \left(2 t^{2}+1\right)}{t^{8}}, \quad h_{1}(t)=t-2, \\
h_{2}(t)=t-1, \\
f_{1}(t)=t-3, \quad f_{2}(t)=t-4, \\
h(t, u, v)=\frac{\left(t^{3}+1\right) u^{2} v}{t^{10} \ln (t+2)}, \quad Q_{1}(t)=\frac{2 M+4 M^{3}}{\left(1+t^{3}\right)\left(1+v^{2}\right)}, \\
R_{2}(t)=\frac{\left(1+N^{2}\right)^{2}}{t^{10} \ln (t+2)}, \quad \frac{(t, u, v) \in\left[t_{0},+\infty\right) \times \mathbb{R}^{2} .}{M^{10} \ln (2+t)}, \\
R_{1}(t)=\frac{M^{2}}{\left(1+t^{3}\right)\left(1+t^{2}\right)},
\end{gathered}
$$

It is easy to verify that (16)-(18) and (42) are satisfied. It follows from Theorem 5 that (11) possesses uncountably many bounded positive solutions in $A(N, M)$. On the other hand, for any $L \in(N, M)$, there exist $\theta \in(0,1)$ and $T>t_{0}+$ $|a|+\tau+\left|T_{0}\right|$ such that the Mann iterative sequence $\left\{x_{m}\right\}_{m \in \mathbb{N}_{0}}$ with mixed errors generated by (43) converges to a bounded positive solution $x \in A(N, M)$ of (11) and has the error estimate (22), where $\left\{\gamma_{m}\right\}_{m \in \mathbb{N}_{0}}$ is an arbitrary sequence in 
$A(N, M)$, and $\left\{\alpha_{m}\right\}_{m \in \mathbb{N}_{0}}$ and $\left\{\beta_{m}\right\}_{m \in \mathbb{N}_{0}}$ are any sequences in $[0,1]$ satisfying $(23)$ and $(24)$.

Example 13. Consider the following fourth order nonlinear neutral delay differential equation:

$$
\begin{aligned}
& \left(-e^{t}\left(t^{5}\left(\left(1-t^{7}\right)(x(t)-x(t-\tau))^{\prime}\right)^{\prime}\right)^{\prime}\right)^{\prime} \\
& +\left(\frac{1}{1+t^{6}}\right. \\
& \left.\times\left(x^{2}(t-3) x^{2}(t-1)-\frac{2 x^{3}(t+1)}{1+\sqrt{1+x^{2}(t-3)}}\right)\right)^{\prime} \\
& +\frac{1}{3 t^{4}+2 t}\left(x^{3}(t-4)(1+\sin x(2 t-3))\right. \\
& =\frac{\ln (1+t)}{1+t^{10}}, \quad t \geq 4,
\end{aligned}
$$

where $\tau>0, t_{0}=T_{0}=4, k=3, N=100, M=200$, $a=\min \{4-\tau, 0\}$, and

$$
\begin{aligned}
& \alpha(t)=-e^{t}, \quad \beta(t)=t^{5}, \quad \gamma(t)=1-t^{7}, \\
& p(t)=-1, \quad g(t)=\frac{\ln (1+t)}{1+t^{10}}, \\
& h_{1}(t)=t+1, \quad h_{2}(t)=t-3, \quad h_{3}(t)=t-1, \\
& f_{1}(t)=t-4, \quad f_{2}(t)=2 t-3, \\
& f_{3}(t)=3 t-2 \text {, } \\
& f(t, u, v, w)=\frac{1}{3 t^{4}+2 t}\left(u^{3}(1+\sin v)+\frac{v \cos ^{2}(t-1)}{1+w^{2}}\right), \\
& h(t, u, v, w)=\frac{1}{1+t^{6}}\left(v^{2} w^{2}-\frac{2 u^{3}}{1+\sqrt{1+v^{2}}}\right), \\
& Q_{1}(t)=\frac{1}{3 t^{4}+2 t}\left(M^{2}(6+M)+\frac{1+3 M^{2}}{\left(1+N^{2}\right)^{2}} \cos ^{2}(t-1)\right) \text {, } \\
& Q_{2}(t)=\frac{1}{1+t^{6}} \\
& \times\left(4 M^{3}+\frac{6 M^{2}\left(1+\sqrt{1+M^{2}}\right)}{\left(1+\sqrt{1+N^{2}}\right)^{2}}\right. \\
& \left.+\frac{2 M^{4}}{\left(1+\sqrt{1+N^{2}}\right)^{2} \sqrt{1+N^{2}}}\right) \\
& R_{1}(t)=\frac{1}{3 t^{4}+2 t}\left(2 M^{3}+\frac{M}{1+N^{2}}\right),
\end{aligned}
$$

It is easy to verify that (16), (17), (55), and (56) are satisfied. It follows from Theorem 6 that (11) possesses uncountably many bounded positive solutions in $A(N, M)$. On the other hand, for any $L \in(N, M)$, there exist $\theta \in(0,1)$ and $T>$ $t_{0}+|a|+\tau+\left|T_{0}\right|$ such that the Mann iterative sequence $\left\{x_{m}\right\}_{m \in \mathbb{N}_{0}}$ with mixed errors generated by (57) converges to a bounded positive solution $x \in A(N, M)$ of (11) and has the error estimate (22), where $\left\{\gamma_{m}\right\}_{m \in \mathbb{N}_{0}}$ is an arbitrary sequence in $A(N, M)$, and $\left\{\alpha_{m}\right\}_{m \in \mathbb{N}_{0}}$ and $\left\{\beta_{m}\right\}_{m \in \mathbb{N}_{0}}$ are any sequences in $[0,1]$ satisfying $(23)$ and $(24)$.

Example 14. Consider the following fourth order nonlinear neutral delay differential equation:

$$
\begin{aligned}
& \left(\frac{3+\sin t}{t^{2}}\right. \\
& \times\left(t \ln ^{3} t\right. \\
& \left.\left.\quad \times\left(\frac{t^{5}}{1+\sqrt{t}}\left(x(t)+\frac{4+5 t}{3+2 t} x(t-\tau)\right)^{\prime}\right)^{\prime}\right)^{\prime}\right)^{\prime} \\
& +\left(\frac{\left(1-t^{2}\right) x^{3}(3 t-1) x(t-5)}{(1+t)^{6}+t|x(t-2)|}\right)^{\prime} \\
& +\frac{t^{3} x^{3}(t-\sin t)-t^{5} x^{2}(t-\cos t)}{1+t^{11}+x^{2}(t-22)}=\frac{t^{3}}{1+t^{8}}, \\
& +t \geq 20,
\end{aligned}
$$

where $\tau>0, t_{0}=T_{0}=20, k=3, N=3, M=20, c_{1}=3$, $c_{2}=2, a=\min \{20-\tau,-2\}$, and

$$
\begin{gathered}
\alpha(t)=\frac{3+\sin t}{t^{2}}, \quad \beta(t)=t \ln ^{3} t, \quad \gamma(t)=\frac{t^{5}}{1+\sqrt{t}}, \\
p(t)=\frac{4+5 t}{3+2 t}, \quad g(t)=\frac{t^{3}}{1+t^{8}}, \\
h_{1}(t)=3 t-1, \quad h_{2}(t)=t-5, \quad h_{3}(t)=t-2, \\
f_{1}(t)=t-\sin t, \quad f_{2}(t)=t-\cos t,
\end{gathered}
$$




$$
\begin{gathered}
f_{3}(t)=t-22, \quad f(t, u, v, w)=\frac{t^{3} u^{3}-t^{5} v^{2}}{1+t^{11}+w^{2}} \\
h(t, u, v, w)=\frac{\left(1-t^{2}\right) u^{3} v}{(1+t)^{6}+t|w|}
\end{gathered}
$$

$Q_{1}(t)$

$$
\begin{gathered}
=\frac{3\left(t^{3}+t^{14}\right) M^{2}+5 t^{3} M^{4}+2\left(t^{5}+t^{16}\right) M+4 t^{5} M^{3}}{\left(1+t^{11}+N^{2}\right)^{2}}, \\
Q_{2}(t)=\frac{4\left(1+t^{2}\right)(1+t)^{6} M^{3}+5 t\left(1+t^{2}\right) M^{4}}{\left[(1+t)^{6}+t N\right]^{2}}, \\
R_{1}(t)=\frac{t^{3} M^{3}+t^{5} M^{2}}{1+t^{11}+N^{2}}, \\
R_{2}(t)=\frac{\left(1+t^{2}\right) M^{4}}{(1+t)^{6}+t N}, \quad(t, u, v, w) \in\left[t_{0},+\infty\right) \times \mathbb{R}^{3} .
\end{gathered}
$$

It is easy to verify that (16)-(18), (71), and (72) are satisfied. It follows from Theorem 7 that (11) possesses uncountably many bounded positive solutions in $A(N, M)$. On the other hand, for any $L \in\left(\left(c_{1} / c_{2}\right) M+c_{1} N,\left(c_{2} / c_{1}\right) N+c_{2} M\right)$, there exist $\theta \in(0,1)$ and $T>t_{0}+|a|+\tau+\left|T_{0}\right|$ such that the Mann iterative sequence $\left\{x_{m}\right\}_{m \in \mathbb{N}_{0}}$ with mixed errors generated by (73) converges to a bounded positive solution $x \in A(N, M)$ of (11) and has the error estimate (22), where $\left\{\gamma_{m}\right\}_{m \in \mathbb{N}_{0}}$ is an arbitrary sequence in $A(N, M)$, and $\left\{\alpha_{m}\right\}_{m \in \mathbb{N}_{0}}$ and $\left\{\beta_{m}\right\}_{m \in \mathbb{N}_{0}}$ are any sequences in $[0,1]$ satisfying $(23)$ and $(24)$.

Example 15. Consider the following fourth order nonlinear neutral delay differential equation:

$$
\left(\frac { \operatorname { l n } t } { t } \left(\frac { t ^ { 2 } } { 2 + \operatorname { c o s } t } \left(\frac{t \ln ^{2}(1+t)}{1+\sin ^{2} t}\right.\right.\right.
$$

$$
\begin{gathered}
\left.\left.\left.\times(x(t)+(-3-\sin t) x(t-\tau))^{\prime}\right)^{\prime}\right)^{\prime}\right)^{\prime} \\
+\left(\frac{(1-\sqrt{t}) x^{2}(t-5) x^{2}(\sqrt{t}-7)}{1+t^{3}+\sin ^{2}(t x(t-3))}\right)^{\prime} \\
+\frac{2-x^{2}(\sqrt{t})-x^{2}(t-1) x^{3}(t-10)}{1+t^{5}+\ln \left(t^{2}+3\right)} \\
=\frac{3+\cos \left(t^{2}+1\right)}{t^{5}+2 t^{3}}, \quad t \geq 9
\end{gathered}
$$

where $\tau>0, t_{0}=T_{0}=9, k=3, N=10, M=50, c_{1}=4$, $c_{2}=2, a=\min \{9-\tau,-4\}$, and

$$
\begin{aligned}
& \alpha(t)=\frac{\ln t}{t}, \quad \beta(t)=\frac{t^{2}}{2+\cos t}, \\
& \gamma(t)=\frac{t \ln ^{2}(1+t)}{1+\sin ^{2} t}, \quad p(t)=-3-\sin t, \\
& g(t)=\frac{3+\cos \left(t^{2}+1\right)}{t^{5}+2 t^{3}}, \quad h_{1}(t)=t-5, \\
& h_{2}(t)=\sqrt{t}-7, \quad h_{3}(t)=t-3, \\
& f_{1}(t)=\sqrt{t}, \quad f_{2}(t)=t-1, \quad f_{3}(t)=t-10, \\
& h(t, u, v, w)=\frac{(1-\sqrt{t}) u^{2} v^{2}}{1+t^{3}+\sin ^{2}(t w)}, \\
& f(t, u, v, w)=\frac{2-u^{2}-v^{2} w^{3}}{1+t^{5}+\ln \left(t^{2}+3\right)}, \\
& Q_{1}(t)=\frac{2 M+5 M^{4}}{1+t^{5}+\ln \left(t^{2}+3\right)}, \\
& Q_{2}(t)=\frac{2 M^{3}(1+\sqrt{t})\left(2 t^{3}+4+t M\right)}{\left(1+t^{3}\right)^{2}}, \\
& R_{1}(t)=\frac{2+M^{2}+M^{5}}{1+t^{5}+\ln \left(t^{2}+3\right)}, \\
& R_{2}(t)=\frac{(1+\sqrt{t}) M^{4}}{1+t^{3}}, \quad(t, u, v, w) \in\left[t_{0},+\infty\right) \times \mathbb{R}^{3} .
\end{aligned}
$$

It is easy to verify that (16)-(18) and (82) are satisfied. It follows from Theorem 8 that (11) possesses uncountably many bounded positive solutions in $A(N, M)$. On the other hand, for any $L \in\left(\left(c_{1}-1\right) N,\left(c_{2}-1\right) M\right)$, there exist $\theta \in(0,1)$ and $T>t_{0}+|a|+\tau+\left|T_{0}\right|$ such that the Mann iterative sequence $\left\{x_{m}\right\}_{m \in \mathbb{N}}$ with mixed errors generated by (83) converges to a bounded positive solution $x \in A(N, M)$ of (11) and has the error estimate (22), where $\left\{\gamma_{m}\right\}_{m \in \mathbb{N}_{0}}$ is an arbitrary sequence in $A(N, M)$, and $\left\{\alpha_{m}\right\}_{m \in \mathbb{N}_{0}}$ and $\left\{\beta_{m}\right\}_{m \in \mathbb{N}_{0}}$ are any sequences in $[0,1]$ satisfying $(23)$ and $(24)$.

\section{Conflict of Interests}

The authors declare that there is no conflict of interests regarding the publication of this paper.

\section{Acknowledgment}

The first author was supported by the Science Research Foundation of Educational Department of Liaoning Province (L2012380). The third author was supported by Basic Science Research Program through the National Research Foundation of Korea (NRF) funded by the Ministry of Science, ICT and Future Planning (2013R1A1A2057665). 


\section{References}

[1] R. P. Agarwal and S. R. Grace, "Oscillation theorems for certain neutral functional differential equations," Computers and Mathematics with Applications, vol. 38, no. 11, pp. 1-11, 1999.

[2] Á. Elbert, "Oscillation/Nonoscillation criteria for linear second order differential equations," Journal of Mathematical Analysis and Applications, vol. 226, no. 1, pp. 207-219, 1998.

[3] C. Huang, "Oscillation and nonoscillation for second order linear differential equations," Journal of Mathematical Analysis and Applications, vol. 210, no. 2, pp. 712-723, 1997.

[4] M. R. S. Kulenović and S. Hadžiomerspahić, "Existence of nonoscillatory solution of second order linear neutral delay equation," Journal of Mathematical Analysis and Applications, vol. 228, no. 2, pp. 436-448, 1998.

[5] X. Lin, "Oscillation of second-order nonlinear neutral differential equations," Journal of Mathematical Analysis and Applications, vol. 309, no. 2, pp. 442-452, 2005.

[6] L. S. Liu, "Ishikawa and mann iterative process with errors for nonlinear strongly accretive mappings in banach spaces," Journal of Mathematical Analysis and Applications, vol. 194, no. 1, pp. 114-125, 1995.

[7] Z. Liu and S. M. Kang, "Infinitely many nonoscillatory solutions for second order nonlinear neutral delay differential equations," Nonlinear Analysis, Theory, Methods and Applications, vol. 70, no. 12, pp. 4274-4293, 2009.

[8] H. Z. Qin, N. N. Shang, and Y. M. Lu, "A note on oscillation criteria of second order nonlinear neutral delay differential equations," Computers and Mathematics with Applications, vol. 56, no. 12, pp. 2987-2992, 2008.

[9] X. H. Tang and Y. Liu, "Bounded oscillation for second-order delay differential equations with unstable type in a critical case," Applied Mathematics Letters, vol. 16, no. 3, pp. 263-268, 2003.

[10] Y. H. Yu and H. Z. Wang, "Nonoscillatory solutions of secondorder nonlinear neutral delay equations," Journal of Mathematical Analysis and Applications, vol. 311, no. 2, pp. 445-456, 2005.

[11] Q. G. Yang, L. J. Yang, and S. M. Zhu, "Interval criteria for oscillation of second-order nonlinear neutral differential equations," Computers and Mathematics with Applications, vol. 46, no. 5-6, pp. 903-918, 2003.

[12] Y. Zhou, "Existence for nonoscillatory solutions of secondorder nonlinear differential equations," Journal of Mathematical Analysis and Applications, vol. 331, no. 1, pp. 91-96, 2007.

[13] S. M. Kang, Z. Liu, L. Chen, and S. Y. Cho, "Existence of nonoscillatory solutions for a third-order nonlinear neutral delay differential equation," Abstract and Applied Analysis, vol. 2011, Article ID 693890, 23 pages, 2011. 


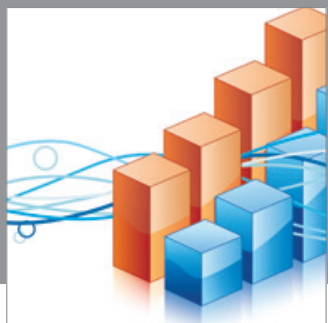

Advances in

Operations Research

mansans

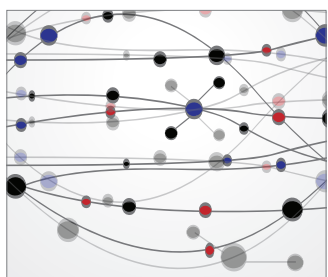

The Scientific World Journal
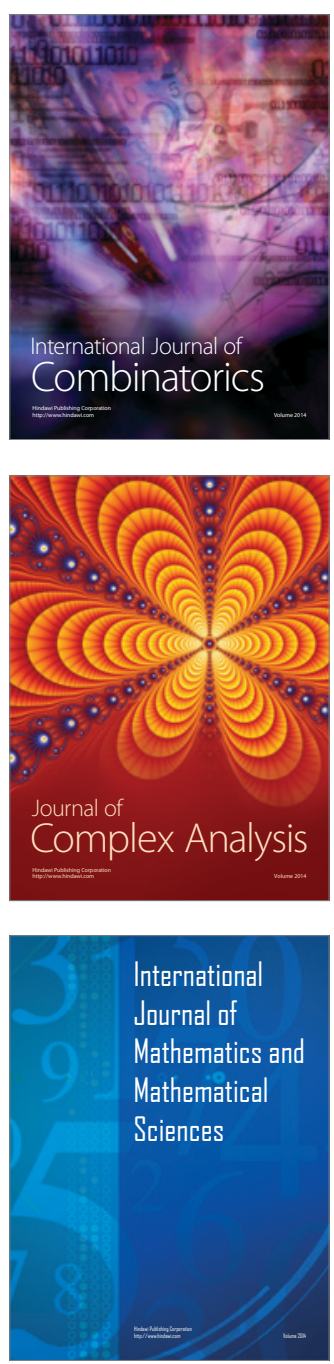
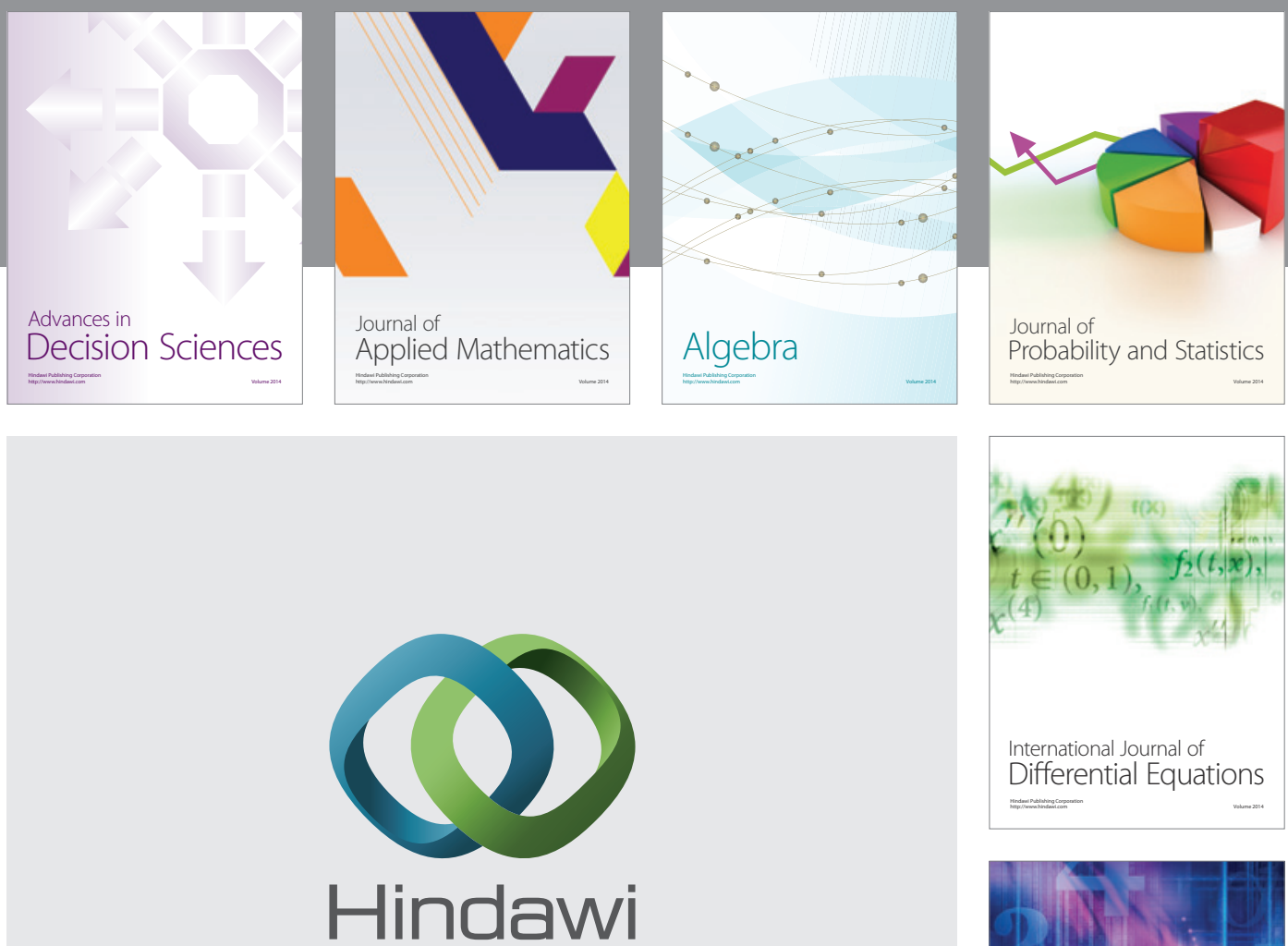

Submit your manuscripts at http://www.hindawi.com
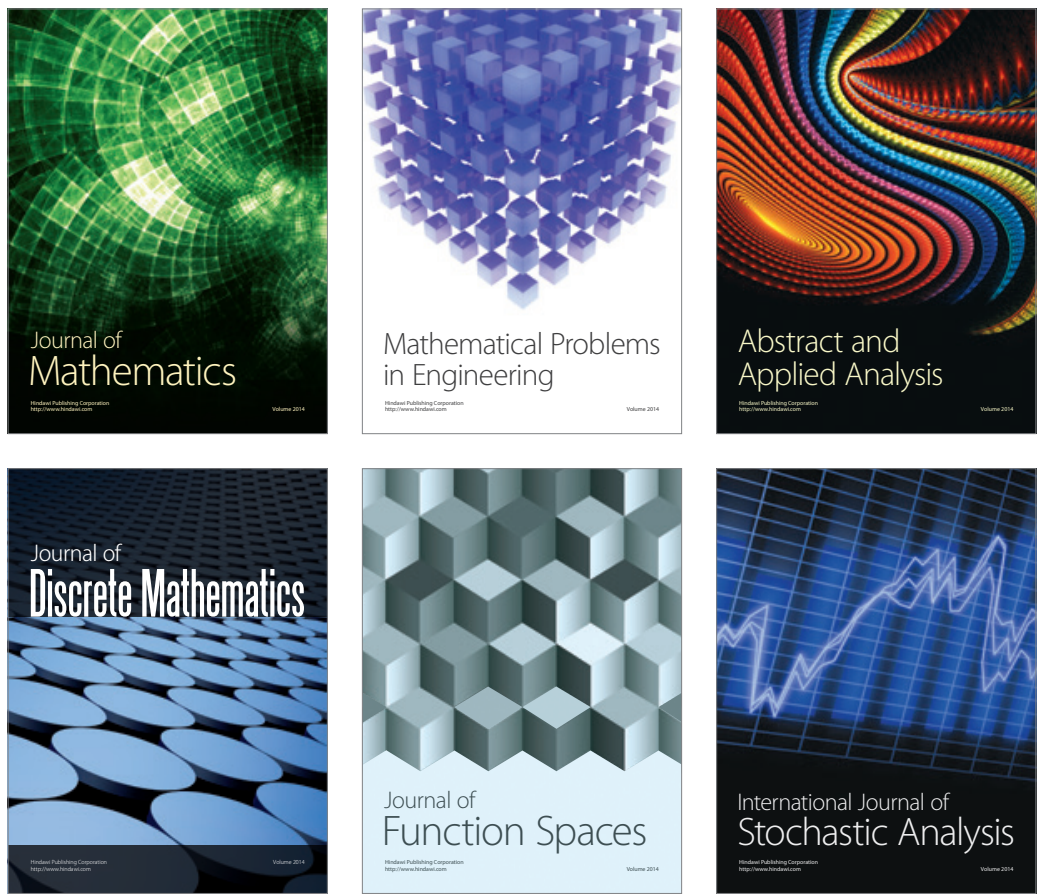

Journal of

Function Spaces

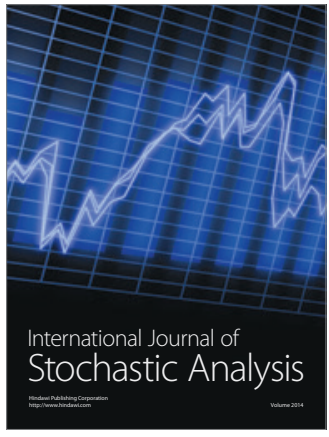

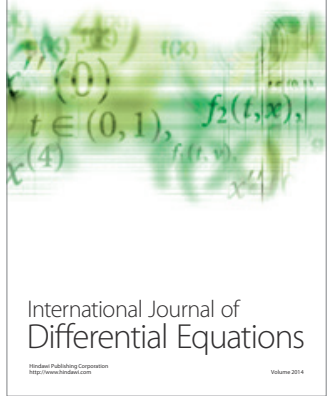
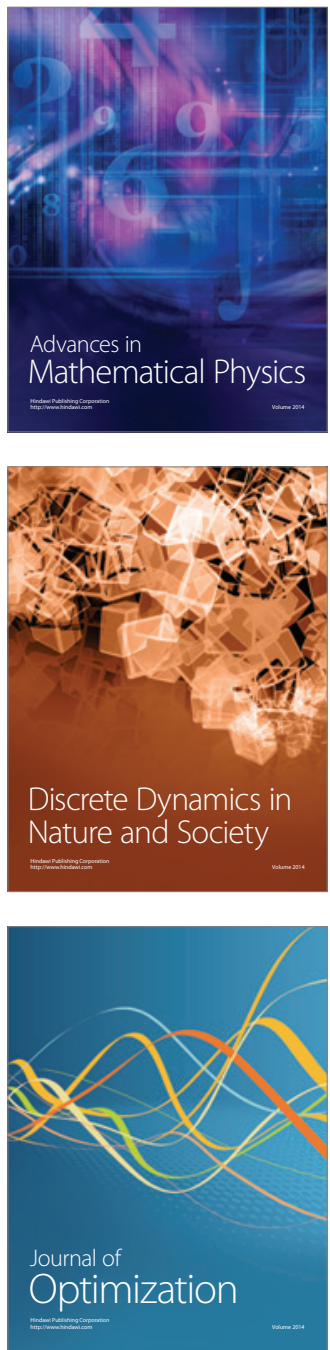